This item was submitted to Loughborough's Research Repository by the author.

Items in Figshare are protected by copyright, with all rights reserved, unless otherwise indicated.

\title{
A numerical study of the effects of loading from diffusive deposition on the efficiency of fibrous filters
}

PLEASE CITE THE PUBLISHED VERSION

http://dx.doi.org/10.1016/j.jaerosci.2005.08.001

PUBLISHER

(C) Elsevier

VERSION

AM (Accepted Manuscript)

LICENCE

CC BY-NC-ND 4.0

REPOSITORY RECORD

Dunnett, Sarah J., and Charles F. Clement. 2012. "A Numerical Study of the Effects of Loading from Diffusive Deposition on the Efficiency of Fibrous Filters”. figshare. https://hdl.handle.net/2134/11249. 
This item was submitted to Loughborough's Institutional Repository (https://dspace.lboro.ac.uk/) by the author and is made available under the following Creative Commons Licence conditions.

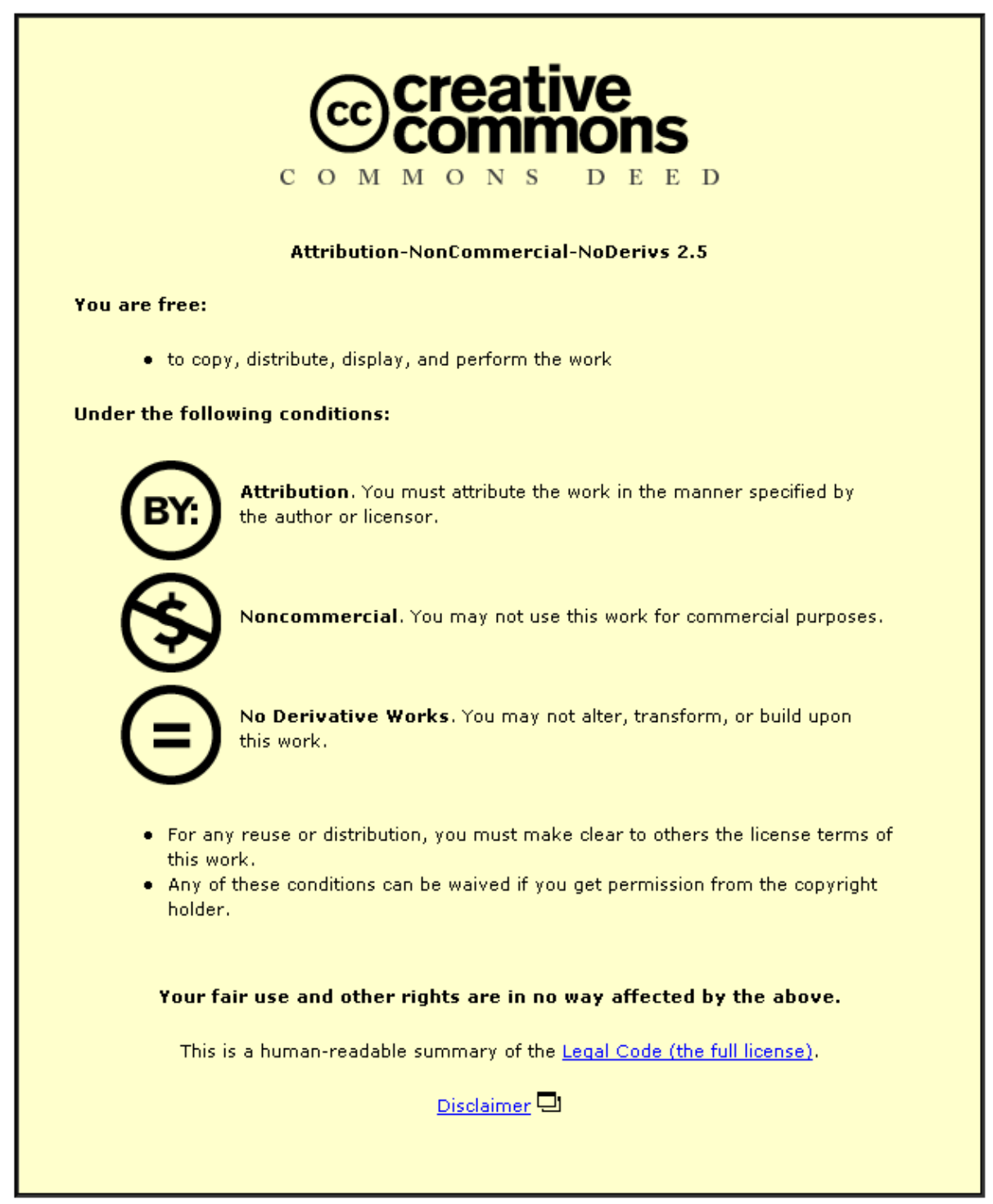

For the full text of this licence, please go to: http://creativecommons.org/licenses/by-nc-nd/2.5/ 


\section{A NUMERICAL STUDY OF THE EFFECTS OF LOADING FROM DIFFUSIVE DEPOSITION ON THE EFFICIENCY OF FIBROUS FILTERS

\author{
S.J.Dunnett ${ }^{1 *}$, C.F.Clement ${ }^{2}$ \\ ${ }^{1}$ Department of Aeronautical and Automotive Engineering, Loughborough University \\ , Loughborough, Leics. LE11 3TU, U.K. \\ 2 \\ 15 Witan Way, Wantage, Oxon OX12 9EU, U.K.
}

Running title: A numerical study of fibrous filtration

* Corresponding author. 


\section{Nomenclature}

$A_{\kappa}, B_{\kappa}$
$C_{\kappa}, D_{\kappa}$ Functions of $\kappa$ used in the analytical solution of $\left[\frac{\mathrm{dn}}{\mathrm{dr}}\right]_{\mathrm{r}=1+\kappa, \theta=0}$

$b_{0} \quad$ Dimension of the cell enclosing the fibre used in flow model.

D Diffusion Coefficient

d Fibre width

E variable used in the analytical solution of $\left[\frac{\mathrm{dn}}{\mathrm{dr}}\right]_{\mathrm{r}=1+\kappa, \theta=0}\left(=\mathrm{C}_{\kappa} / \delta^{3}\right)$

F exponent function used in the analytical solution of $\left[\frac{\mathrm{dn}}{\mathrm{dr}}\right]_{\mathrm{r}=1+\kappa, \theta=0}$

g function of $\theta$ describing the new surface of the fibre containing deposit.

I integral

k Hydrodynamic factor used in the flow model of Stechkina and Fuchs (1966)

$\mathrm{N} \quad$ Normal to the fibre surface

$\mathrm{N}_{\mathrm{C}}$ number of segments the boundary of the solution domain is divided into when solving for the flow field.

n Particle concentration

$\mathrm{n}_{0} \quad$ Particle concentration in the undisturbed flow

Pe Peclet number

Re Reynolds number of the flow

r Polar coordinate

$\mathrm{r}_{\mathrm{p}} \quad$ Particle radius

$\mathrm{r}_{1} \quad$ interception radius $\left(=\mathrm{d} / 2+\mathrm{r}_{\mathrm{p}}\right)$

$\mathrm{U}_{0} \quad$ Fluid (air) velocity

$\mathrm{U} \quad$ Fluid velocity component in the $\mathrm{x}$ direction

$\mathrm{V} \quad$ Fluid velocity component in the y direction

$\mathrm{U}_{\mathrm{r}} \quad$ Fluid velocity component in the $\mathrm{r}$ direction

$\mathrm{U}_{\theta} \quad$ Fluid velocity component in the $\theta$ direction

$\mathrm{X}, \mathrm{Y}$ integration variables used in the analytical solution of $\left[\frac{\mathrm{dn}}{\mathrm{dr}}\right]_{\mathrm{r}=1+\kappa, \theta=0}$

x,y Cartesian components

$\alpha \quad$ Packing fraction 
$\beta \quad$ Constant describing the rate at which particles deposit upon the fibre surface

$\gamma \quad$ Integration variable used in the analytical solution of $\left[\frac{\mathrm{dn}}{\mathrm{dr}}\right]_{\mathrm{r}=1+\kappa, \theta=0}$

$\delta \quad$ Non-dimensional thickness of the diffusion layer

$\lambda$ Constant related to the rate at which particles deposit upon the fibre surface $\left(=\beta[\partial \mathrm{n} / \partial \mathrm{N}]_{\theta=0}\right)$

$\eta \quad$ Particle collection efficiency

$\eta_{\mathrm{D}} \quad$ Contribution to $\eta_{\mathrm{SF}}$ from deposition

$\eta_{\mathrm{SF}} \quad$ Particle collection efficiency as defined by Stechkina and Fuchs (1966)

$\eta_{\kappa} \quad$ Contribution to $\eta_{\mathrm{SF}}$ from interception

$\kappa \quad$ Ratio of particle and fibre radii

$\mu \quad$ Air viscosity

$\theta \quad$ Polar coordinate

$\rho \quad$ Density of air

$\omega \quad$ Fluid vorticity

$\psi \quad$ Stream function 


\begin{abstract}
This work is aimed at developing numerical methods for calculating diffusive and interceptive deposition on fibres in fibrous filters as a deposit builds up. Calculations are performed of the two dimensional flow field past a single fibre for three different cell models using the boundary element model (BEM). Boundary conditions for the cells correspond to the Kuwabara model and two different rectangular cases, one corresponding to a periodic cell array. The concentration field for particles is then calculated using the equation of Stechkina and Fuchs (1966), but with the numerical flow field. Resulting deposition rates are compared with their results and also with those of Friedlander (1977) for point particles. For deposition on the front of the fibre, we extend their results using the analytic flow field to obtain analytic results for parameter regions where different types of deposition occur. The two relevant parameters are the ratio of the particle to fibre radii and the effective thickness of the diffusion layer. Numerical flow fields are used to calculate particle deposition over the whole fibre, assuming the deposit forms as a smooth solid layer. The new surface shape is parameterised, and the whole process of recalculating the flow field and particle deposition is repeated. Results are obtained for deposition on the new surface as functions of flow Peclet number and fibre packing fraction.
\end{abstract}

Keywords: filtration; fibrous filters; numerical simulation 


\section{Introduction}

Filtration by fibrous filters is one of the simplest and most effective ways to separate particulate matter from fluid and filters find diverse application. For example, many employees in industry or agriculture need to avoid exposure to airborne particulates and protection is usually provided by face masks or larger scale filtration units. Also medical and biological fields rely on clean air and filtration can improve air quality in all areas of life.

A fibrous filter consists of many threadlike fibres, of varying sizes, positioned more or less normal to the direction of the fluid flow. The fluid passes through the regions between the fibres and particles which are suspended in the fluid are removed by their collision and attachment to the fibre surface. The particle deposit builds up on the fibres and in the limit clogging occurs and further filtration simply results in cake formation at the filter surface which necessitates filter replacement or regeneration.

In order to understand the performance of fibrous filters it is necessary to obtain a description of the fluid flow and much work has been undertaken in this area. As the structure of the fibrous filters is complex, simplified models have been developed. Generally the assumption is made that the Reynolds number of the flow is small and hence the inertia of the flow is neglected. Many of the earlier works have then adopted 'cell' models where the equations of the fluid flow are solved in a two dimensional 'cell', surrounding the single fibre, where the boundary conditions take into account neighbouring fibres, see Kuwabara (1959), Happel (1959). These models have been widely adopted to model the fluid flow. Other approaches have been adopted, see for example Brown (1984) who determined the stream function that gives rise to the lowest rate of dissipation of energy by viscous drag. Numerical techniques such as the Boundary Element Method, see Hildyard et al (1985) and control volume method, see Rao and Faghri (1988) and Dhaniyala and Liu (1999) have also been adopted to determine the flow field. Once a description of the flow field through the filter is known the transport of the particles by the flow and their possible removal by the fibres can be investigated. Much research has been undertaken into understanding the removal of particles by the mechanisms of interception, inertial impaction, gravitational capture and diffusional deposition for a 
clean filter and is now reasonably well understood. See for example Lee and Gieseke (1980), Fardi and Lui (1992), Ingham et al (1989), Harrop and Stenhouse (1969), Pich (1973), Stechkina and Fuchs (1966), Zhu et al (2000)., Asgharian and Chenh (2002). However the feedback effects of the particle deposition upon subsequent deposition and filter performance are less well understood and this is the situation considered in this paper. Experimental investigations in this area include Japuntich et al (1994) and Thomas et al (2001) where the penetration and pressure drop of various filters were considered under loading. Modelling work which has been undertaken in this area includes the semi-empirical models of fibre efficiency as a function of deposit, see Stenhouse et al (1992) and Stenhouse and Trottier (1991). These models are restricted to specific cases and based on limited experimental data. The models of dendrite growth by Payatakes and colleagues, Payatakes et al (1977), Payatakes and Gradon (1980), which are limited due to the neglect of the effects of the dendrite upon the flow field. The theoretical studies of Konstandopoulos et al (1998) and Kostoglou and Konstandopoulos (2000) considered the shape evolution of particulate deposits on cylinders. In the paper by Konstandopoulos et al (1998) the case of inertial deposition on an isolated cylinder in potential flow was studied. The Boundary Element Method was adopted to compute the flow past the cylinder with and without deposition. In the paper of Kostoglou and Konstandopoulos (2000) the deposit evolution on a cylinder was considered for inertial impaction in the limit of high Stokes number. Other studies which attempt to take into account the change in the flow field due to the particle deposit are restricted due to their considerable use of computing time and resources, see Jung and Tien (1993), Biggs et al (2003), Karadimos and Ocone (2003) and Przekop et al (2003).

In the work described here cell models have been adopted to determine the flow field using the Boundary Element Method. This numerical method has the advantage of only needing to discretise the domain boundary in order to solve for the flow field, hence requiring significantly lower computing resources than other available approaches. The same approach was adopted by Konstandopoulos et al (1998), however they considered particles suspended in potential flow and the mechanism of deposition was inertial impaction, representative of a cylinder at the front of a filter. In this work slow flow has been assumed and the deposition on the fibres due to diffusion has then been considered for various situations representative of fibres 
throughout the filter, using three different cell models. Considering the change in the shape of the boundary due to the deposition, the flow field and hence particle deposition has been recalculated. The effects upon subsequent deposition and efficiency are then investigated. As the only mechanism by which particles are assumed to deposit is diffusion the model developed here is only valid for particles whose size is a lot smaller than the size of the fibres. Also, in the model developed here, the particle deposit is assumed to form a smooth solid layer whose width is a function of position on the fibre. This allows for the feedback effects from the deposition upon further deposition and filter efficiency to be investigated. However it does mean that the complex dendrite structures which do form on the surface of fibres have not been modelled accurately and hence the results obtained give a qualitative description of the effect of deposition.

Overall the present work is intended to provide a practical means of calculating deposition in individual cells of a cell model as a function of the cell parameters and particle deposition. Realistically there is considerable variation in these parameters for an actual filter and the results will be used in a statistical model, currently under development, which takes account of these variations and which lead to a distribution of the efficiency with which a single cell removes particles. 


\section{Fluid flow formulation}

In this work we are considering infinitely long cylinders with the air flow moving perpendicular to the cylinder axis. Hence the flow is two dimensional and the motion takes place in the plane perpendicular to the cylinder axis. Generally for fluid flow through a filter the Reynolds number, Re, of the flow is small, where Re is defined by, $\operatorname{Re}=\frac{\mathrm{d} \rho \mathrm{U}_{0}}{\mu}$, $\mathrm{d}$ is the diameter of the fibres making up the filter, $\rho$ is the density of the air, $\mathrm{U}_{0}$ is the air velocity and $\mu$ the air viscosity. In this case the flow is described by the biharmonic equation for the stream function $\psi$, i.e.

$$
\nabla^{4} \psi=0
$$

In the work described here equation (1) was solved using the Boundary Element Method (BEM). In order to apply this method equation (1) is first reduced to its coupled equivalent form,

$$
\begin{aligned}
& \nabla^{2} \psi=\omega \\
& \nabla^{2} \omega=0
\end{aligned}
$$

where $\omega$ is the fluid vorticity which is a measure of the tendency of the fluid to rotate or to cause rotation of suspended bodies during flow.

Consider the general two dimensional domain $\mathrm{S}$ bounded by the contour $\mathrm{C}$. By using the Rayleigh-Green biharmonic boundary formula (see Jaswon and Symm (1977)) and Greens second identity we may obtain the equivalent pair of coupled integral equations (Kelmanson (1983a))

$$
\begin{aligned}
& \chi(\underline{p}) \psi(\underline{p})=\int_{C}\left\{\psi(\underline{q}) G_{1}{ }^{\prime}(\underline{p}, \underline{q})-\psi^{\prime}(\underline{q}) G_{1}(\underline{p}, \underline{q})+\omega(\underline{q}) G_{2}^{\prime}(\underline{p}, \underline{q})-\omega^{\prime}(\underline{q}) G_{2}(\underline{p}, \underline{q})\right\} d q \\
& \chi(\underline{p}) \omega(\underline{p})=\int_{C}\left\{\omega(\underline{q}) G_{1}{ }^{\prime}(\underline{p}, \underline{q})-\omega^{\prime}(\underline{q}) G_{1}(\underline{p}, \underline{q})\right\} d q
\end{aligned}
$$

where

(i) $\mathrm{p} \in \mathrm{S} \cup \mathrm{C}, \underline{\mathrm{q}} \in \mathrm{C}$

(ii) A prime denotes differentiation with respect to the outward normal to $\mathrm{S}$ at $\mathrm{q} \in \mathrm{C}$.

(iii) dq is the differential increment of $\mathrm{C}$ at $\mathrm{q} \in \mathrm{C}$. 
(iv) $\chi(\underline{p})$ is defined by :

$$
\chi(\underline{p})=\left\{\begin{array}{l}
0 \quad \text { if } \underline{p} \notin S \cup C \\
2 \pi \text { if } \underline{p} \in S \\
\text { Internal angle included between the tangents to } C \text { on either side of } \underline{p} \text { if } \underline{p} \in C
\end{array}\right\}
$$

(v) $G_{1}(\underline{p}, \underline{q})=\ln |\underline{p}-\underline{q}|, \quad G_{2}(\underline{p}, \underline{q})=\frac{1}{4}|\underline{p}-\underline{q}|^{2}\{\ln |\underline{p}-\underline{q}|-1\}$

In order to solve equations (3) for $\psi$ and $\omega$ at the general point $\underline{\mathrm{p}} \in \mathrm{S} \cup \mathrm{C}$ we need a complete set of boundary information for $\psi, \psi^{\prime}, \omega, \omega^{\prime}$. The physical boundary conditions of viscous flow give only two of these equations at each point $\underline{\mathrm{q}} \in \mathrm{C}$. In order to obtain a complete set of information on the boundary, equations (3) are applied at a point $\underline{\mathrm{q}}_{1} \in \mathrm{C}$ to obtain:

$$
\begin{aligned}
& \int_{\mathrm{C}}\left\{\psi(\underline{\mathrm{q}}) \mathrm{G}_{1}{ }^{\prime}\left(\underline{\mathrm{q}}_{1}, \underline{\mathrm{q}}\right)-\psi^{\prime}(\underline{\mathrm{q}}) \mathrm{G}_{1}\left(\underline{\mathrm{q}}_{1}, \underline{\mathrm{q}}\right)+\omega(\underline{\mathrm{q}}) \mathrm{G}_{2}^{\prime}\left(\underline{\mathrm{q}}_{1}, \underline{\mathrm{q}}\right)-\omega^{\prime}(\underline{\mathrm{q}}) \mathrm{G}_{2}\left(\underline{\mathrm{q}}_{1}, \underline{\mathrm{q}}\right)\right\} \mathrm{dq} \\
& -\chi\left(\underline{\mathrm{q}}_{1}\right) \psi\left(\underline{\mathrm{q}}_{1}\right)=0 \\
& \int_{\mathrm{C}}\left\{\omega(\underline{\mathrm{q}}) \mathrm{G}_{1}{ }^{\prime}\left(\underline{\mathrm{q}}_{1}, \underline{\mathrm{q}}\right)-\omega^{\prime}\left(\underline{\mathrm{q}}^{\mathrm{q}}\right) \mathrm{G}_{1}\left(\underline{\mathrm{q}}_{1}, \underline{\mathrm{q}}\right)\right\} \mathrm{dq}-\chi\left(\underline{\mathrm{q}}_{1}\right) \omega\left(\underline{\mathrm{q}}_{1}\right)=0
\end{aligned}
$$

where $\underline{\mathrm{q}}_{\mathrm{q}_{1}} \in \mathrm{C}$

Equations (4) can be solved to give the remaining boundary information at each point $\underline{\mathrm{q}}_{1} \in \mathrm{C}$. Having obtained a complete set of boundary information equations (3) can be used to generate $\psi$ and $\omega$ at any point in the solution domain.

In order to solve equations (4) and subsequently equations (3), numerical methods generally must be adopted. The procedure which has been adopted is to divide the boundary $C$ into a number, $\mathrm{N}_{\mathrm{C}}$, of straight line segments $\mathrm{C}_{1}---\mathrm{C}_{\mathrm{N}}$, such that

$$
\mathrm{C}=\bigcup_{\mathrm{j}=1}^{\mathrm{N}_{\mathrm{C}}} \mathrm{C}_{\mathrm{j}}
$$

On each of the $C_{j}$ it is assumed that $\psi, \psi^{\prime}, \omega$ and $\omega^{\prime}$ have the piecewise constant value of $\psi_{j}, \psi_{j}^{\prime}, \omega_{j}$ and $\omega_{j}^{\prime}$ respectively, which are their values at the midpoints of the segments. A discretised form of equations (4) (Kelmanson (1983b)) is then applied at the midpoint $\underline{q}=\underline{q}_{i}, i=1---N_{C}$ of each interval. This generates a set of $2 N_{C}$ linear simultaneous equations in the $2 \mathrm{~N}_{\mathrm{C}}$ unknowns. Solution of these equations gives the complete set of boundary information. A discretised form of equation (3) is then 
applied at the general point $\mathrm{p} \in \mathrm{S} \cup \mathrm{C}$ to obtain $\psi(\mathrm{p})$ and $\omega(\mathrm{p})$. Having obtained $\psi$ it is then possible to obtain the velocities of the flow from

$\mathrm{U}=\frac{\partial \psi}{\partial \mathrm{y}}, \mathrm{V}=-\frac{\partial \psi}{\partial \mathrm{x}}$

In the work presented here $\psi$ has been obtained for the two different domains shown in Figs 1a-b. Before making any calculations all quantities were non-dimensionalised, distances with respect to fibre radius, $d / 2$, and velocities with respect to the mean flow velocity, $\mathrm{U}_{0}$. The form of equation (3) is unchanged.

The domain shown in Fig. 1a is equivalent to the 'cell' studied by Kuwabara (1959) and models a system of parallel, staggered, cylinders directed normal to the flow. The 'cell' consists of a surface concentric with the fibre surface at such a distance that the packing fraction of the fibre within this cylinder is identical to that of the fibres within the filter as a whole. The packing fraction, $\alpha$, is defined as the fraction of the perceived volume of the filter that is actually occupied by fibres The physical boundary conditions are that there is no flow across the fibre surface and on the outer boundary the flow is moving with the mean flow velocity $\mathrm{U}_{0}$. In terms of $\psi$ and $\omega$ these conditions are:

(a) $\psi=0, \omega=0$ on $\mathrm{AB}$ and $\mathrm{CD}$

(b) $\psi=0, \psi^{\prime}=0$ on $\mathrm{BC}$

(c) $\psi=\mathrm{h}_{1} \sin \gamma, \omega=0$ on DA

where $\gamma$ is the angle made with the positive $x$ axis and $h_{1}$ is given by $h_{1}=1 / \sqrt{\alpha}$. This model will be called model 1 in this work.

The domain shown in Fig. 1b models a system of parallel cylindrical fibres such that the axes of the cylinders are parallel and perpendicular to the airflow. The cell in this case is the area defined by ADEF. Two different sets of boundary conditions have been applied to this domain corresponding to two physically different set-ups. In both cases there is no flow across the surface of the fibres or across the symmetry plane AD leading to the conditions, in dimensionless units

(a) $\psi=\mathrm{h}_{2}, \omega=0$ on AB, DC

(b) $\psi=\mathrm{h}_{2}, \psi^{\prime}=0$ on BC

(c) $\psi=0, \omega=0$ on EF. 
In model 2 the cylinders are assumed to be staggered as in model 1 and the extra boundary conditions taken are:

(d) $\psi=\mathrm{h}_{2}+\mathrm{y}, \omega=0$ on AF, DE

In model 3 the cylinder are not staggered but are positioned such that their axes lie in the same plane. The extra boundary conditions taken are:

(d) $\psi^{\prime}=0, \omega^{\prime}=0$ on AF, DE

For models 2 and 3 the packing fraction, $\alpha$, is given by $\alpha=\pi / 4 \mathrm{~h}^{2}$.

For all 3 models the flow velocities were determined at any position in the domains shown using the Boundary Element method described.

\section{Particle motion.}

Neglecting the influence of electric effects the main mechanisms by which point particles are captured by the fibres within the filter are

(i) inertial impaction, when particles deviate from the streamlines of the air flow due to their inertia and impact upon the fibres

(ii) gravitational settling, when particles settle out of the air moving through the filter due to the influence of gravity.

(iii) diffusional deposition, in which the combined effect of the air motion and the Brownian motion of the particle brings it into contact with a fibre.

Taking into account the finite size of particles of radius $r_{p}$ these mechanisms are augmented by the interception of the particles with the fibres, see Fig. 2 where the mechanism is shown for a clean fibre. Mechanisms (i) and (ii) are generally applicable for larger particles and mechanism (iii) for smaller particles. In this work we are considering the deposition of smaller particles as these are particles for which filters are generally used in aerosol science. Hence mechanism (iii) is the case we are considering and our method takes account of interception of particles with fibres by using the appropriate boundary conditions. For steady state conditions the particle concentration is described by equation (11), see Stechkina and Fuchs (1966).

$\mathrm{U}_{\mathrm{r}} \frac{\partial \mathrm{n}}{\partial \mathrm{r}}+\frac{\mathrm{U}_{\theta}}{\mathrm{r}} \frac{\partial \mathrm{n}}{\partial \theta}=\mathrm{D}\left(\frac{\partial^{2} \mathrm{n}}{\partial \mathrm{r}^{2}}+\frac{1}{\mathrm{r}} \frac{\partial \mathrm{n}}{\partial \mathrm{r}}+\frac{1}{\mathrm{r}^{2}} \frac{\partial^{2} \mathrm{n}}{\partial \theta^{2}}\right)$

where $U_{r}$ and $U_{\theta}$ are the components of air velocity in polar coordinates, $n$ is the particle concentration and D is the coefficient of diffusion of the particles. Non- 
dimensionalising distances with respect to fibre radius, velocities with respect to $U_{0}$, and $\mathrm{n}$ with respect to $\mathrm{n}_{0}$, the particle concentration in the undisturbed flow. Equation (11) then becomes

$$
\mathrm{U}_{\mathrm{r}} \frac{\partial \mathrm{n}}{\partial \mathrm{r}}+\frac{\mathrm{U}_{\theta}}{\mathrm{r}} \frac{\partial \mathrm{n}}{\partial \theta}=\frac{2}{\mathrm{Pe}}\left(\frac{\partial^{2} \mathrm{n}}{\partial \mathrm{r}^{2}}+\frac{1}{\mathrm{r}} \frac{\partial \mathrm{n}}{\partial \mathrm{r}}+\frac{1}{\mathrm{r}^{2}} \frac{\partial^{2} \mathrm{n}}{\partial \theta^{2}}\right)
$$

where

$$
\mathrm{Pe}=\frac{\mathrm{U}_{0} \mathrm{~d}}{\mathrm{D}}
$$

is the Peclet number, which is a measure of the relative magnitude of the diffusional motion of the particles and the convective motion of the air past the fibre. All quantities in equation (12) are now dimensionless. For fibrous filtration the third term on the right hand side of equation (12) is much smaller than the other terms and hence has been neglected.

Therefore the problem of determining the particle concentration in the vicinity of a fibre reduces to solving

$$
\mathrm{U}_{\mathrm{r}} \frac{\partial \mathrm{n}}{\partial \mathrm{r}}+\frac{\mathrm{U}_{\theta}}{\mathrm{r}} \frac{\partial \mathrm{n}}{\partial \theta}=\frac{2}{\operatorname{Pe}}\left(\frac{\partial^{2} \mathrm{n}}{\partial \mathrm{r}^{2}}+\frac{1}{\mathrm{r}} \frac{\partial \mathrm{n}}{\partial \mathrm{r}}\right)
$$

subject to the boundary conditions that $n=1$ away from the fibre and $n=0$ at the interception radius, which is $1+\kappa$ for a clean fibre, where $\kappa=2 r_{p} / d$ is the ratio of the particle and fibre radii. For large Peclet numbers the particles diffuse to the fibre from a thin layer (boundary layer) adjacent to its surface. The thickness of this layer is much less than half the distance between the fibres, which gives the condition that $\delta<<1 / \sqrt{\alpha}$ where $\delta$ is the non-dimensional thickness of the diffusion layer. In order to solve equation (14) it is necessary to apply a third boundary condition. This was done in a similar way to Stechkina and Fuchs (1966). Along the line $\theta=0, \mathrm{U}_{\theta}=0$, and hence equation (14) reduces to an ordinary differential equation

$$
\frac{2}{\mathrm{Pe}} \frac{\mathrm{d}^{2} \mathrm{n}}{\mathrm{dr}^{2}}+\frac{\mathrm{dn}}{\mathrm{dr}}\left(\frac{2}{\mathrm{r} \cdot \mathrm{Pe}}-\mathrm{U}_{\mathrm{r}}\right)=0
$$

Dividing the line $\theta=0$ into $n-1$ segments the derivatives can be approximated using finite differences and the value of $U_{r}$ determined at any point using the BEM described earlier. This generated a set of equations for $n$ at the ends of the segments which can then be solved, hence giving the values of $\mathrm{n}$ at finite points along $\theta=0$. 
Equation (14) was then solved numerically using the finite difference approach. A grid was generated by taking $\mathrm{m}$ radial lines emanating from the fibre centre and $\mathrm{k}$ curves parallel to the fibre surface. Initially for the clean filter these will be concentric circles but as the shape of the fibre surface changes due to particle deposition these gridlines will also change. The domain in which the grid was generated was taken so that any increase in its size had no significant effect upon the results obtained. The partial derivatives in equation (14) were then approximated using the values of $n$ at the grid points, the $n_{i j}$, for $i=1, k, j=1$, $m$, by finite difference approximations and a system of equations for the $n_{i j}$ generated. These were then solved to give the value of $\mathrm{n}$ at all the grid points. Various values of $\mathrm{k}$ and $\mathrm{m}$ were considered until any further increase in their values had no significant effect upon the results obtained. If an expression is available for $U_{r}$ along $\theta=0$ then equation (15) can be treated analytically. Such a treatment has also been undertaken here by approximating $U_{\mathrm{r}}$, and is described in the next section. This investigation gives us a better understanding of particle deposition upon the fibre for different physical conditions.

\section{Analytic solution of equation (15)}

In this section, we extend the analytic treatment of eq.(15) by Stechkina and Fuchs (1966) to obtain expressions for the deposition flux, $\mathrm{dn} / \mathrm{dr}$, at $\theta=0$. The parameters controlling the flux are related to the deposition geometry and particle variables, and we find different expressions in different parameter regions.

Following Stechkina and Fuchs (1966), the non-dimensional radial flow field in (15) is taken to be a form valid near the cylinder surface:

$$
\mathrm{U}_{\mathrm{r}}=\frac{\left(1-\frac{1}{\mathrm{r}^{2}}-2 \ln \mathrm{r}\right)}{2 \mathrm{k}}
$$

where the hydrodynamic factor, for an isolated cylinder, is given by the Lamb equation in terms of the flow Reynolds number, $\mathrm{k}=2-\ln$ Re.

For a system of parallel cylinders (model 1), we use the expression in terms of the packing fraction, $\alpha$,

$$
\mathrm{k}=-\frac{1}{2} \ln (\alpha)-\frac{3}{4}+\alpha-\frac{\alpha^{2}}{4}
$$

Equation (15) can now be integrated, as by Stechkina and Fuchs (1966), to obtain 
$\left[\frac{\mathrm{dn}}{\mathrm{dr}}\right]_{\mathrm{r}=1+\kappa, \theta=0}=\frac{\mathrm{I}^{-1}}{(1+\kappa)}$

where the integral is

$$
\begin{gathered}
I=\int_{1+\kappa}^{\infty}(\mathrm{dr} / \mathrm{r}) \exp \left\{[3(\mathrm{r}-1-\kappa)+1 / \mathrm{r}-1 /(1+\kappa)-2 \mathrm{r} \ln \mathrm{r}+2(1+\kappa) \ln (1+\kappa)] / \delta^{3}\right\} \\
=\int_{0}^{\infty}[\mathrm{d} \gamma /(1+\kappa+\gamma)] \exp \{[3 \gamma+1 /(1+\kappa+\gamma)-1 /(1+\kappa)-2(1+\kappa+\gamma) \ln (1+\kappa+\gamma) \\
\left.+2(1+\kappa) \ln (1+\kappa)] / \delta^{3}\right\},
\end{gathered}
$$

where the integration variable has been changed to $\gamma=r-1-\kappa$.

The quantity, $\delta$, which represents the thickness of the diffusion layer around the cylinder, is given by

$\delta=\left(\frac{4 \mathrm{k}}{\mathrm{Pe}}\right)^{1 / 3}$

The integral, I, is a function of the two parameters, $\delta$ and $\kappa$, and it can be shown that, for the range of values of $\delta$ and $\kappa$ that we need to consider, the exponent in the integrand becomes exceedingly small well before $\gamma=1$. The exponent in the integrand, which we term $\mathrm{F}(\gamma, \kappa, \delta)$, can then be expanded in a series in $\gamma /(1+\kappa)$. Because the first two terms in the expansion vanish when $\kappa=0$, to cover all physical cases it has been found necessary in general to go to $4^{\text {th }}$ order:

$\mathrm{F}(\gamma, \kappa, \delta)=\left[-\mathrm{A}_{\kappa}(\kappa) \gamma-\mathrm{B}_{\kappa}(\kappa) \gamma^{2}-\mathrm{C}_{\kappa}(\kappa) \gamma^{3}+\mathrm{D}_{\kappa}(\kappa) \gamma^{4}\right] / \delta^{3}$,

Where

$$
\begin{aligned}
& \mathrm{A}_{\kappa}(\kappa)=2 \ln (1+\kappa)-\frac{\kappa(\kappa+2)}{(1+\kappa)^{2}} \approx 2 \kappa^{2}-\frac{10 \kappa^{3}}{3} \\
& \mathrm{~B}_{\kappa}(\kappa)=\frac{\kappa(\kappa+2)}{(1+\kappa)^{3}} \approx 2 \kappa-5 \kappa^{2} \\
& \mathrm{C}_{\kappa}(\kappa)=\frac{1}{(1+\kappa)^{2}}\left(\frac{1}{(1+\kappa)^{2}}-\frac{1}{3}\right) \approx \frac{2}{3} \\
& \mathrm{D}_{\kappa}(\kappa)=\frac{1}{(1+\kappa)^{3}}\left(\frac{1}{(1+\kappa)^{2}}-\frac{1}{6}\right) \approx \frac{5}{6}
\end{aligned}
$$


To bring out the complex behaviour of this expansion, the coefficients have been expanded for small $\kappa$. Both the coefficients $A_{\kappa}$ and $B_{\kappa}$ vanish in the point particle limit when $\kappa=0$. The dominant falloff in the exponential may be provided by either one of the first three terms depending on the values of $\kappa$ and $\delta$, the fourth term always being a relatively small correction. To understand which term is dominant in the exponent, $F$, we change the integration variable to $X=A_{\kappa} \gamma / \delta^{3}$, or $X^{2}=$ $\mathrm{B}_{\kappa} \gamma^{2} / \delta^{3}$, when the integral takes the following alternative forms:

$$
\begin{gathered}
\mathrm{I}=\left(\delta^{3} / \mathrm{A}_{\kappa}\right) \int_{0}^{\infty}\left[\mathrm{dX} /\left(1+\kappa+\delta^{3} \mathrm{X} / \mathrm{A}_{\kappa}\right)\right] \exp \left[-\mathrm{X}-\mathrm{B}_{\kappa} \delta^{3} \mathrm{X}^{2} / \mathrm{A}_{\kappa}{ }^{2}-\mathrm{C}_{\kappa} \delta^{6} \mathrm{X}^{3} / \mathrm{A}_{\kappa}{ }^{3}\right. \\
\left.-\mathrm{D}_{\kappa} \delta^{9} \mathrm{X}^{4} / \mathrm{A}_{\kappa}{ }^{4}\right]
\end{gathered}
$$

or

$$
\begin{gathered}
\mathrm{I}=\left(\delta^{3 / 2} / \mathrm{B}_{\kappa}{ }^{1 / 2}\right) \int_{0}^{\infty}\left[\mathrm{dX} /\left(1+\kappa+\delta^{3 / 2} \mathrm{X} / \mathrm{B}_{\kappa}{ }^{1 / 2}\right)\right] \exp \left[-\mathrm{A}_{\kappa} \mathrm{X} /\left(\mathrm{B}_{\kappa} \delta^{3}\right)^{1 / 2}-\mathrm{X}^{2}-\mathrm{C}_{\kappa} \delta^{3 / 2} \mathrm{X}^{3} / \mathrm{B}_{\kappa}{ }^{3 / 2}\right. \\
\left.-\mathrm{D}_{\kappa} \delta^{3} \mathrm{X}^{4} / \mathrm{B}_{\kappa}{ }^{2}\right]
\end{gathered}
$$

The boundaries between the region where the $\mathrm{X}$ term provides the exponential cutoff and the regions where the $\mathrm{X}^{2}$ or $\mathrm{X}^{3}$ term provide the cutoff are points for which the coefficients of these terms in the first exponent are equal to 1 , giving the critical values of $\delta$ :

$\delta_{\mathrm{A}-\mathrm{B}}=\left(\mathrm{A}_{\kappa}^{2} / \mathrm{B}_{\mathrm{K}}\right)^{1 / 3}, \quad \delta_{\mathrm{A}-\mathrm{C}}=\left(\mathrm{A}_{\mathrm{K}}^{3} / \mathrm{C}_{\mathrm{K}}\right)^{1 / 6}$.

Similarly, from the second exponent, the boundary between the region where the $\mathrm{X}^{2}$ term provides the exponential cutoff and the region where the $\mathrm{X}^{3}$ term provides the cutoff is given by:

$\delta_{\mathrm{B}-\mathrm{C}}=\mathrm{B}_{\mathrm{K}} / \mathrm{C}_{\mathrm{K}}^{2 / 3}$.

In Figure 3 we show the results of calculations of the $\delta_{\mathrm{A}-\mathrm{B}}$ and $\delta_{\mathrm{B}-\mathrm{C}}$ boundaries in the $\kappa-\delta$ plane compared to points in the plane calculated for the physical examples given in Table 1. The $\mathrm{X}$ cutoff dominates in the bottom region shown in figure 3 and the $\mathrm{X}^{3}$ cutoff in the top region. In the narrow intermediate region, through which runs the $\delta_{\mathrm{A} \text {-C }}$ boundary which we have not plotted, the $\mathrm{X}^{2}$ term is the largest in the exponent, although it is not generally possible to neglect the other terms. In the extreme regions, one term in the exponent is dominant and we can obtain analytic approximations to the integral. Accurate boundaries where these approximations are valid have not been determined so that the neglected terms may make sizeable contributions to the integral at points near to the boundaries. For the first small $\delta$ region, it is easy to calculate resulting corrections to the main result as we now show. 


\section{The lower, small $\delta$, region}

Here $A_{\kappa}$ is the largest term in the exponent, and we expand the $B_{\kappa}$ and $C_{K}$ terms and the $(1+\kappa+\gamma)^{-1}$ term to first order in $\gamma$ to obtain

$$
\begin{aligned}
I & =(1+\kappa)^{-1} \int_{0}^{\infty} d \gamma\left[1-\gamma /(1+\kappa)-\gamma^{2} B_{\kappa}(\kappa) / \delta^{3}-\gamma^{3} C_{\kappa}(\kappa) / \delta^{3}\right] \exp \left[-A_{\kappa}(\kappa) \gamma / \delta^{3}\right] \\
& =(1+\kappa)^{-1}\left[\delta^{3} / A_{\kappa}-\left(\delta^{3} / A_{\kappa}\right)^{2} /(1+\kappa)-2\left(B_{\kappa} / \delta^{3}\right)\left(\delta^{3} / A_{\kappa}\right)^{3}\right. \\
& \left.-6\left(C_{\kappa} / \delta^{3}\right)\left(\delta^{3} / A_{\kappa}\right)^{4}\right] .
\end{aligned}
$$

\section{The upper, larger $\delta$, region}

Here, the $\mathrm{C}_{\mathrm{K}}$ term in the exponent dominates and the simplest approximation is to take first,

$\mathrm{I}=(1+\kappa)^{-1} \int_{0}^{\infty} \mathrm{d} \gamma \exp \left(-\mathrm{E} \gamma^{3}\right)$

where

$\mathrm{E}=\mathrm{C}_{\mathrm{\kappa}}(\kappa) / \delta^{3}$.

We change the variable to $\mathrm{Y}=\mathrm{E}^{1 / 3} \gamma$ when the integral becomes

$\mathrm{I}=\mathrm{E}^{-1 / 3}(1+\kappa)^{-1} \mathrm{I}_{0}$

where the dimensionless integral is a constant:

$\mathrm{I}_{0}=\int_{0}^{\infty} \mathrm{dY} \exp \left(-\mathrm{Y}^{3}\right)=2 \sqrt{3} \pi /[9 \Gamma(2 / 3)]=0.8930$.

In terms of the original parameters,

$\mathrm{I}=0.8930 \mathrm{C}_{\kappa}(\kappa)^{-1 / 3} \delta(1+\kappa)^{-1}$.

Values of the integral I given in equation (20) were also determined numerically for various values of $\kappa$ and $\delta$ and were found to be in good agreement with the analytical expressions (28) and (32).

To return to the comparison with the physical data of Table 1 shown in Figure 3, we see that the physical points spread across from the upper region, where the $C_{k}$ term dominates, to the lower region, where the $A_{\kappa}$ term dominates, as $\kappa$ increases. Case 6 of $\kappa=0.5$ is a rather unphysical case of a particle half as wide as the fibre which is well into region $A_{\kappa}$. However, for very small values of $\delta$, particles of much smaller values of $\kappa$ will also be in this region, and presumably have a similar type of deposition pattern. The point particle limit of $\kappa=0$ does not extend far into larger $\kappa$ when $\delta$ is very small. 
Values of $\delta$ are consistently smaller for parallel fibres than for isolated or random fibres. The flux at $\theta=0$ given by the integral will be smaller in this geometry, resulting from the shadowing of particle flux by the preceding fibre. Altogether, evaluation of the parameters $\delta$ and $\kappa$, should help in the characterization of particle deposition. 


\section{Results}

a) Clean filter

Initially the case of a clean filter was considered. This is the situation most often considered in the past literature and hence there are results available with which to validate the model described here. An important characteristic used to evaluate the performance of a filter is the particle collection efficiency, $\eta$, which is the measure of the efficiency of a single representative fibre in the filter. This is defined to be the ratio of the number of particles that are actually removed by the fibre to the number entering the 'cell'. Hence, in terms of dimensional quantities,

$$
\eta=\frac{2 \mathrm{D} \int_{\theta=0}^{\theta=\pi}\left[\frac{\partial \mathrm{n}}{\partial \mathrm{N}}\right]_{\mathrm{r}=\mathrm{r}_{1}} \mathrm{ds}}{2 \mathrm{U}_{0} \mathrm{~b}_{0} \mathrm{n}_{0}}
$$

where $r_{1}$ is the interception radius which will change as deposition of particles occurs, $\mathrm{b}_{0}$ is the dimension of the cell and $\mathrm{N}$ is the normal to the fibre surface. In terms of dimensionless quantities equation (33) becomes

$$
\eta=\frac{2 \mathrm{I} \sqrt{\alpha}}{\mathrm{Pe}}
$$

where I is the integral

$$
\mathrm{I}=\int_{\theta=0}^{\theta=\pi}\left[\frac{\partial \mathrm{n}}{\partial \mathrm{N}}\right]_{\mathrm{r}=\mathrm{r}_{1}} \mathrm{ds}
$$

where $\mathrm{n}, \mathrm{N}, \mathrm{r}_{1}$ and ds in equation (35) are all dimensionless. In the case of the clean fibre this integral is relatively straightforward to determine numerically with $d s=r_{1} d \theta$. Initially, in order to compare with the work of Stechkina and Fuchs (1966) we define an efficiency, $\eta_{\mathrm{SF}}$, to be the ratio of the number of particles that are actually removed by the fibre to the number crossing an area equal to that of the fibre. This can be written as:

$$
\eta_{\mathrm{SF}}=\frac{2 \mathrm{D}\left(\mathrm{a}_{0}+\mathrm{r}_{\mathrm{p}}\right) \int_{0}^{\pi}\left[\frac{\partial \mathrm{n}}{\partial \mathrm{r}}\right]_{\mathrm{r}=\mathrm{r}_{1}} \mathrm{~d} \theta}{2 \mathrm{U}_{0} \mathrm{a}_{0} \mathrm{n}_{0}}
$$

In terms of dimensionless quantities, equation (36) becomes

$$
\eta_{\mathrm{SF}}=\frac{2(1+\kappa) \int_{0}^{\pi}\left[\frac{\partial \mathrm{n}}{\partial \mathrm{r}}\right]_{\mathrm{r}=\mathrm{r}_{1}} \mathrm{~d} \theta}{\mathrm{Pe}}
$$


Values of $\eta_{\text {SF }}$ have been determined for various values of $\kappa$, Pe and packing fraction $\alpha$. Results are shown in Figure 4, where $\eta_{\text {SF }}$ is shown as a function of Pe for $\alpha=0.05$. In obtaining these results, model 1 was adopted in the flow calculations, this is the same system as considered by Stechkina and Fuchs (1966) and hence results obtained using their formula for $\eta_{\mathrm{SF}}$ are also shown. Their formula was developed from their numerical results and given by:

$\eta_{\mathrm{SF}}=\eta_{\mathrm{D}}+\eta_{\kappa}+\frac{1.24 \kappa^{2 / 3}}{\sqrt{\mathrm{k} \cdot \mathrm{Pe}}}$

where:

$\eta_{\mathrm{D}}=2.9 \mathrm{k}^{-1 / 3} \mathrm{Pe}^{-2 / 3}+\frac{0.624}{\mathrm{Pe}}$

$\eta_{\kappa}=\frac{(1+\kappa) \ln (1+\kappa)}{\mathrm{k}}-\frac{1+\kappa}{2 \mathrm{k}}+\frac{1}{2 \mathrm{k}(1+\kappa)}$

and $\mathrm{k}$ is given by equation (18). As can be seen in the figures the results obtained here agree well with Stechkina and Fuchs (1966) work, other values of $\alpha$ were considered and similar results were obtained with agreement best for small $\alpha$, this is expected as Stechkina and Fuchs (1966) model is only valid for small $\alpha$.

It appears therefore that the method adopted is modelling the situation well, although calculations for large $\kappa$ are not realistic because of the relatively large size of the particles.

Considering the effect the packing fraction $\alpha$ has upon efficiency figure 5 shows $\eta$ plotted as a function of Pe for $\kappa=0.0025$ for 3 different values of $\alpha, 0.01,0.05$ and 0.1 using model 1 . As can be seen $\eta$ increases with $\alpha$, this is to be expected as increasing $\alpha$ leads to an increase in the proportion of space in a cell occupied by the fibre and its diffusion layer. The smaller Peclet numbers correspond to lower flow velocities which give particles more time to diffuse to the fibre. The main purpose of this current work is to investigate the effects of particle deposition on the fibres upon subsequent deposition. As deposition is a function of $\partial \mathrm{n} / \partial \mathrm{N}$ on the fibre surface, where $\mathrm{N}$ is the normal to the surface, this normal derivative is a quantity of interest in our calculations. In figure $6 \partial \mathrm{n} / \partial \mathrm{N}$ is shown as a function of $\theta$, where $\theta=0$ corresponds to the front of the fibre and $\theta=\pi$ the rear, for $\alpha=0.05$. Results are shown for the three numerical models, models 1-3, described for the air flow. Also shown is the 
expression derived by Friedlander (1977) for $\partial \mathrm{n} / \partial \mathrm{N}$ for point particles. The behaviour of the derivative was investigated for various physical situations and it was found to fall into two regimes when using models 1 and 2 . In one case $\partial \mathrm{n} / \partial \mathrm{N}$ has an initial very gradual decrease from its maximum value at $\theta=0$ and a more rapid decrease to zero near $\theta=\pi$. In the other case $\partial \mathrm{n} / \partial \mathrm{N}$ has a fairly rapid decrease at the front of the fibre which then tails off to zero at the rear. These two regimes correspond to the different regions identified by the analytical solution described earlier. An example of each case is shown in fig. 6 where in fig. $6 a \mathrm{Pe}=5891$ and $\kappa=0.0025$ which corresponds to small $\kappa$, large $\delta$, and in fig. $6 \mathrm{~b} \mathrm{Pe}=363114, \kappa=0.05$ which corresponds to larger $\kappa$, smaller $\delta$. The results in fig. 6a correspond to the physical conditions, $d=40 \mu \mathrm{m}, \mathrm{U}_{0}=0.1 \mathrm{~m} / \mathrm{s}$ and $\mathrm{r}_{\mathrm{p}}=50 \mathrm{~nm}$ and those in fig. $6 \mathrm{~b}$ to $\mathrm{d}=20 \mu \mathrm{m}, \mathrm{U}_{0}=0.5 \mathrm{~m} / \mathrm{s}$ and $\mathrm{r}_{\mathrm{p}}=500 \mathrm{~nm}$. As can be seen, for both cases, models 1 and 2 give similar results but model 3 gives a smaller value of $\partial \mathrm{n} / \partial \mathrm{N}$ at the front of the fibre. In model 3 the fibres are not taken to be staggered but assumed to lie in-line, hence symmetry on the flow field has been imposed up and down stream of the fibre. This leads to the differences shown in the results in figure 6.

The expression derived by Friedlander is for point particles and hence is only valid as $\kappa \rightarrow 0$ which explains the bad agreement with the numerical results in fig. $6 \mathrm{~b}$, however agreement is good in fig. $6 a$ where $\kappa$ is small. The two regimes for the behaviour of $\partial \mathrm{n} / \partial \mathrm{N}$ as $\theta$ increases can be explained physically. Away from the immediate vicinity of the fibre, particles are carried by the flow but in the thin diffusion layer adjacent to the fibre, particles diffuse to the surface. The nondimensional thickness of this layer, $\delta$, can be approximated by equation (21). The behaviour of $\partial \mathrm{n} / \partial \mathrm{N}$ as $\theta$ increases shown in fig. 6a corresponds to relatively large values of $\delta$, small $\kappa$ and hence deposition due to diffusion is dominant and interception is not significant. The behaviour shown in fig. $6 \mathrm{~b}$ corresponds to conditions such that the non-dimensional radius of the particle is of the same order, or larger, than $\delta$. In this case interception will also have a significant affect upon deposition. In the rest of this work the first case will be considered, i.e. large $\delta$, small 
$\kappa$. In this case, for staggered fibres, it is possible to approximate $\partial \mathrm{n} / \partial \mathrm{N}$ as a function of $\theta$ by the expression

$$
\frac{\partial \mathrm{n}}{\partial \mathrm{N}}=\left[\frac{\partial \mathrm{n}}{\partial \mathrm{N}}\right]_{\theta=0}\left(1-\mathrm{a} \theta^{\mathrm{p}}\right)^{\mathrm{b}}
$$

where a, b and p are constants determined by the least squares method. Examples of how well equation (40) fits the numerical results are shown in Figure 7 for (a) $\mathrm{Pe}=1473, \kappa=0.05$, (b) $\mathrm{Pe}=5891, \kappa=0.0025$, (c) $\mathrm{Pe}=14728, \kappa=0.005$. These correspond to (a) $\mathrm{r}_{\mathrm{p}}=50 \mathrm{~nm}, \mathrm{~d}=2 \mu \mathrm{m}$ and $\mathrm{U}_{0}=0.5 \mathrm{~m} / \mathrm{s}$, (b) $\mathrm{r}_{\mathrm{p}}=50 \mathrm{~nm}, \mathrm{~d}=40 \mu \mathrm{m}$ and $\mathrm{U}_{0}=0.1 \mathrm{~m} / \mathrm{s}$ and (c) $r_{p}=50 \mathrm{~nm}, d=20 \mu \mathrm{m}$ and $U_{0}=0.5 \mathrm{~m} / \mathrm{s}$. The numerical results shown in Fig. 7 were obtained using model 1 for the flow field. 
b) Loaded filter

In the following section the effect deposition has upon subsequent filter performance is investigated. In obtaining the results shown model 1 has been adopted in order to solve for the flow field though either of the other methods could equally as well be applied.

Using equation (40) it is possible to determine the boundary of the new surface formed when particles have deposited on the fibres. As the rate at which particles deposit is proportional to $\partial \mathrm{n} / \partial \mathrm{N}$ it is possible to write the equation of the new surface as $\mathrm{r}=1+\beta \frac{\partial \mathrm{n}}{\partial \mathrm{N}}=1+\lambda\left(1-\mathrm{a} \theta^{\mathrm{p}}\right)^{\mathrm{b}}$

The term $\partial \mathrm{n} / \partial \mathrm{N}$ is the derivative of $\mathrm{n}$ with respect to the outward normal to the surface, N. For a clean fibre $\mathrm{N}$ is in the radial direction but when particles have deposited this is no longer the case, an explanation on how $\partial \mathrm{n} / \partial \mathrm{N}$ was evaluated here is given in appendix A. The constant $\lambda$, which is a function of time and initial particle concentration, defines the thickness of the layer of deposit on the front of the fibre where $\theta=0$.

Once the new surface formed by the deposit has been described it is possible to recalculate the flow field using the BEM method described earlier. The conditions $\psi=\mathrm{h}, \psi^{\prime}=0$ now being imposed on the surface described by equation (41). Having obtained the flow field equations (14) and (15) are solved to obtain the particle concentration in the vicinity of the fibre.

An example of the feedback effect of deposition upon $\partial \mathrm{n} / \partial \mathrm{N}$ is shown in Figure 8 where $\partial \mathrm{n} / \partial \mathrm{N}$ on the surface is shown as a function of $\theta$ when $\alpha=0.05$ for the cases of (i) clean fibre, (ii) one layer of deposit of the fibre and (iii) two layers of deposit on the fibre. In this case a layer is taken to be when the amount of deposit collected on the fibre is such that $\lambda=0.1$. As can be seen from the figure the effects of the initial stages of deposition upon the following deposition distribution on the fibre surface is not great. Hence the particle build up on the fibre surface grows with the majority on the front facing side.

An example of the evolution of the deposit build-up is shown in Figure 9 for $\mathrm{Pe}=5891$, $\kappa=0.0025$, where, as can be seen for the parameters considered, deposit is 
collected over the entire surface with maximum deposition occurring on the front surface. Other values of the parameters have been considered and the distribution of the deposit on the fibre surface is found to be dependent upon Pe and $\kappa$. In the case of small Pe the dominant means by which the particles are transported to the fibre surface is their diffusive motion and hence the distribution of deposit around the fibre surface is more uniform. In the limit of pure diffusion, $\mathrm{Pe}=0$, a uniform distribution is expected. For large Pe convection is the dominant means of transporting particles to the fibre surface and hence particle deposition on the rear of the fibre decreases. Such distributions have been noted previously by Kanaoka et al (1983). The effect of increasing $\kappa$ is also to decrease deposit on the fibre rear as finite size particles intercept with the front surface. For the values of the parameters taken in figure 9 convection is dominant and hence particle deposit is mainly on the front of the fibre although some does deposit on the rear, $\kappa$ is small and hence interception is not playing a significant role. For larger values of $\kappa$ particle deposit does not collect so close to the rear.

Considering the effects that the initial deposition upon the fibre has upon the efficiency of collection, $\eta$, figure 10 shows $\eta$ as a function of Pe for the cases of no deposit and for 1 layer of deposit. Equations (34) and (35) were used to determine $\eta$, an explanation of how the integral, equation (35), was determined for the loaded case is given in appendix $B$. Three different packing fractions are shown, $\alpha=0.01, \alpha=0.05$ and $\alpha=0.1$ and $\kappa=0.0025$. As can be seen, although the effect of the deposition is to decrease $\partial \mathrm{n} / \partial \mathrm{N}$, as shown in figure 8 , the efficiency is actually seen to increase, leading to more deposition. This is due to the greater area over which deposition now takes place as the surface changes. The larger the value of $\alpha$ the greater the effect is. Considering the effects deposition has upon efficiency for different packing fractions, $\alpha$, figure 11 shows $\eta$ as a function of $\alpha$ for the case of no deposit and for 1 layer of deposit for $\mathrm{Pe}=5891, \kappa=0.0025$. As can be seen the effects of deposition upon $\eta$ are more pronounced for the larger values of $\alpha$. 


\section{Conclusions}

In this paper a numerical study has been made of the performance of fibrous filters. In particular the effects of diffusional deposition upon subsequent deposition and filter efficiency have been investigated. In the course of the study is has been shown that the cell model chosen does have a significant effect upon the results obtained with a marked difference been seen in the rate at which particles are deposited upon the staggered and non-staggered fibres. In the case of parallel, staggered fibres when deposition due to diffusion is dominant and interception not significant then the surface formed by the fibre + deposit can be approximated by a simple function of $\theta$. This fact makes it possible, using the numerical technique adopted here, to straightforwardly recalculate the flow field with the deposit on the fibre accounted for. An investigation has then been made into how the deposition affects further deposition and filter efficiency for various packing fractions. As has been observed in previous experimental studies, Thomas et al (2001), filter efficiency was seen to increase as deposition occurs. The larger the value of the packing fraction the more pronounced this effect was seen to be.

The main advantage of the method described in recalculating the flow field following deposition is its simplicity. The BEM only requires the boundary of the solution domain to be divided into segments and as, for the physical conditions considered here, the new surface formed by the deposit can be easily described, this is a straightforward matter. This method of calculation will enable us to evaluate changes to the initial distribution of single fibre efficiencies occurring in a real filter which result from aerosol deposition. 


\section{Appendix A}

The surface of the fibre can be described by the vector

$\underline{\mathrm{r}_{\mathrm{s}}}=((1+\alpha g(\theta)) \cos \theta,(1+\alpha g(\theta)) \sin \theta)$

where

$g(\theta)=\left(1-\mathrm{a}_{1} \theta^{\mathrm{p}_{1}}\right)^{\mathrm{b}_{1}}+\beta_{1}\left(1-\mathrm{a}_{2} \theta^{\mathrm{p}_{2}}\right)^{\mathrm{b}_{2}}+--\beta_{\text {nld }-1}\left(1-\mathrm{a}_{\text {nld }} \theta^{\mathrm{p}_{\text {nld }}}\right)^{\mathrm{b}_{\text {nld }}}$

nld is the number of layers of deposit on the fibre.

The partial derivative of this vector with respect to $\theta$ is then given by

$\frac{\partial \mathrm{r}_{\mathrm{s}}}{\partial \theta}=\left(-(1+\alpha g(\theta)) \sin \theta+\alpha g^{\prime}(\theta) \cos \theta,(1+\alpha g(\theta)) \cos \theta+\alpha g^{\prime}(\theta) \sin \theta\right)$

The vector normal to the surface $\underline{\mathrm{N}}$ will be perpendicular to $\frac{\partial \mathrm{r}_{\mathrm{s}}}{\partial \theta}$ and hence is given by:

$\underline{\mathrm{N}}=\left((1+\alpha g(\theta)) \cos \theta+\alpha g^{\prime}(\theta) \sin \theta,(1+\alpha g(\theta)) \sin \theta-\alpha g^{\prime}(\theta) \cos \theta\right)$

i.e.

$\underline{\mathrm{N}}=\left(\cos \theta+\alpha\left(g(\theta) \cos \theta+\mathrm{g}^{\prime}(\theta) \sin \theta\right), \sin \theta+\alpha\left(g(\theta) \sin \theta-\mathrm{g}^{\prime}(\theta) \cos \theta\right)\right)$

Hence the unit normal $\underline{\hat{N}}$ is given by $\hat{\hat{N}}=N_{x} \underline{i}+N_{y} \underline{j}$ where:

$\mathrm{N}_{\mathrm{x}}=\frac{\cos \theta+\alpha\left(\mathrm{g}(\theta) \cos \theta+\mathrm{g}^{\prime}(\theta) \sin \theta\right)}{|\underline{\mathrm{N}}|}, \mathrm{N}_{\mathrm{y}}=\frac{\sin \theta+\alpha\left(\mathrm{g}(\theta) \sin \theta-\mathrm{g}^{\prime}(\theta) \cos \theta\right)}{|\underline{\mathrm{N}}|}$

and

$|\underline{N}|=\sqrt{1+2 \alpha g(\theta)+\alpha^{2}\left((g(\theta))^{2}+\left(g^{\prime}(\theta)\right)^{2}\right)}$

In terms of polar coordinates $\underline{\hat{N}}=\mathrm{N}_{\mathrm{r}} \underline{\hat{r}}+\mathrm{N}_{\theta} \underline{\hat{\theta}}$ where:

$N_{r}=N_{x} \cos \theta+N_{y} \sin \theta=\frac{1+\alpha g(\theta)}{|\underline{N}|}, N_{\theta}=N_{y} \cos \theta-N_{x} \sin \theta=-\frac{\alpha g^{\prime}(\theta)}{|\underline{N}|}$

Therefore the normal derivative $\frac{\partial \mathrm{n}}{\partial \mathrm{N}}$ can be determined by:

$$
\begin{aligned}
& \frac{\partial \mathrm{n}}{\partial \mathrm{N}}=\nabla \mathrm{n} \cdot \underline{\hat{N}}=\left(\hat{\wedge} \frac{\partial \mathrm{n}}{\partial \mathrm{r}}+\frac{1}{\mathrm{r}} \frac{\partial \mathrm{n}}{\partial \theta} \hat{\theta}\right) \cdot\left(\mathrm{N}_{\mathrm{r}} \hat{\hat{r}}+\mathrm{N}_{\theta} \hat{\hat{\theta}}\right) \\
& =\mathrm{N}_{\mathrm{r}} \frac{\partial \mathrm{n}}{\partial \mathrm{r}}+\frac{\mathrm{N}_{\theta}}{\mathrm{r}} \frac{\partial \mathrm{n}}{\partial \theta}=\frac{(1+\alpha \mathrm{g}(\theta))}{|\underline{\mathrm{N}}|} \frac{\partial \mathrm{n}}{\partial \mathrm{r}}-\frac{\alpha \mathrm{g}^{\prime}(\theta)}{\mathrm{r}|\underline{\mathrm{N}}|} \frac{\partial \mathrm{n}}{\partial \theta}
\end{aligned}
$$


Appendix B

$\mathrm{I}=\int_{\theta=0}^{\theta=\pi}\left[\frac{\partial \mathrm{n}}{\partial \mathrm{N}}\right]_{\mathrm{r}=\mathrm{r}_{1}} \mathrm{ds}=\int_{\theta=0}^{\theta=\pi}\left[\frac{\partial \mathrm{n}}{\partial \mathrm{N}}\right]_{\theta=0, \mathrm{r}=\mathrm{r}_{1}}\left(1-\mathrm{a} \theta^{\mathrm{p}}\right)^{\mathrm{b}} \mathrm{ds}=\left[\frac{\partial \mathrm{n}}{\partial \mathrm{N}}\right]_{\theta=0, \mathrm{r}=\mathrm{r}_{1}} \int_{\theta=0}^{\theta=\pi}\left(1-\mathrm{a} \theta^{\mathrm{p}}\right)^{\mathrm{b}} \mathrm{ds}$

In order to determine this integral ds was determined as a function of $d \theta$

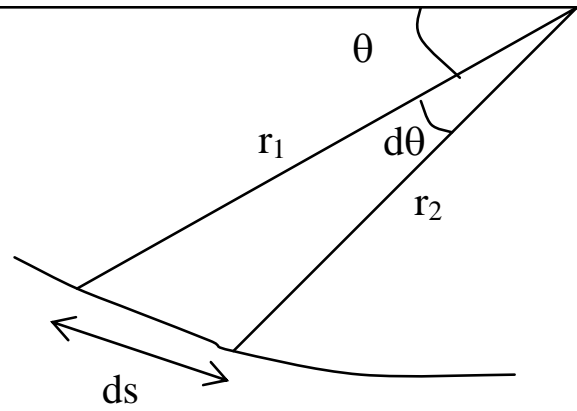

For $\mathrm{d} \theta$ small it can be determined that

$\mathrm{ds}^{2} \approx\left(\mathrm{r}_{1} \mathrm{~d} \theta\right)^{2}+\left(\mathrm{r}_{1}-\mathrm{r}_{2}\right)^{2}$

where

$\mathrm{r}_{1}=1+\kappa+\lambda \mathrm{g}(\theta)$

$\mathrm{r}_{2}=1+\kappa+\lambda \mathrm{g}(\theta+\mathrm{d} \theta)$

If there is one layer of deposit on the fibre then $g(\theta)=\left(1-a_{1} \theta^{p_{1}}\right)^{b_{1}}$, if there are 2 layers $g(\theta)=\left(1-a_{1} \theta^{p_{1}}\right)^{b_{1}}+\beta_{1}\left(1-a_{2} \theta^{p_{2}}\right)^{b_{2}}$ and in general

$g(\theta)=\left(1-\mathrm{a}_{1} \theta^{\mathrm{p}_{1}}\right)^{\mathrm{b}_{1}}+\beta_{1}\left(1-\mathrm{a}_{2} \theta^{\mathrm{p}_{2}}\right)^{\mathrm{b}_{2}}+--\beta_{\text {nld }-1}\left(1-\mathrm{a}_{\text {nld }} \theta^{\mathrm{p}_{\text {nld }}}\right)^{\mathrm{b}_{\text {nld }}}$

where nld is the number of layers of deposit on the fibre.

By assuming that $d \theta$ is small equation $g(\theta)-g(\theta+d \theta)$ can be written as:

$\mathrm{G}(\theta) \mathrm{d} \theta=\mathrm{g}(\theta)-\mathrm{g}(\theta+\mathrm{d} \theta) \approx$

$\mathrm{d} \theta\left[\begin{array}{l}\mathrm{a}_{1} \mathrm{~b}_{1} \mathrm{p}_{1} \theta^{\mathrm{p}_{1}-1}\left(1-\mathrm{a}_{1} \theta^{\mathrm{p}_{1}}\right)^{\mathrm{b}_{1}-1}+\beta_{1} \mathrm{a}_{2} \mathrm{~b}_{2} \mathrm{p}_{2} \theta^{\mathrm{p}_{2}-1}\left(1-\mathrm{a}_{2} \theta^{\mathrm{p}_{2}}\right)^{\mathrm{b}_{2}-1}+-- \\ +\beta_{\text {nld }-1} \mathrm{a}_{\text {nld }} \mathrm{b}_{\text {nld }} \mathrm{p}_{\text {nld }} \theta^{\mathrm{P}_{\text {nld }}-1}\left(1-\mathrm{a}_{\text {nld }} \theta^{\mathrm{p}_{\text {nld }}}\right)^{\mathrm{b}_{\text {nld }}-1}\end{array}\right]$

and hence from (B2)

$\mathrm{ds} \approx \mathrm{d} \theta\left[(1+\kappa+\mathrm{g}(\theta))^{2}+\lambda^{2} \mathrm{G}(\theta)^{2}\right]$

The integral (B1) can then be determined numerically. 


\section{References}

Asgharian, B. and Chenh, Y.S. (2002) The filtration of fibrous aerosol, Aerosol Sci. and tech., 36, pp10-17.

Biggs, M.J., Humby, S.J., Buts, A. and Tuzun, U. (2003) Explicit numerical simulation of suspension flow with deposition in porous media: influence of local flow field variation on deposition processes predicted by trajectory methods, Chem. Eng. Sci., 58, pp1271-1288.

Brown, R.C. (1984) A many-fibre model of airflow through a fibrous filter, J. Aerosol Sci., 5, pp583-593.

Brown, R.C. (1993) Air filtration. An integrated approach to the theory and applications of fibrous filters, Pergamon press, Oxford.

Dhaniyala, S. and Liu, B.Y.H. (1999) An asymmetrical, three-dimensional model for fibrous filters, Aerosol Sci. and tech., 30, pp333-348.

Fardi, B. and Liu, B.Y.H. (1992) Flow field and pressure drop of filters with rectangular fibres, Aerosol Sci. and tech., 17, pp36-44.

Friedlander, S.K. (1977) Smoke, dust and haze. Fundamentals of aerosol behaviour, John Wiley and Sons, New York.

Happel, J. (1959) Viscous flow relative to arrays of cylinders, A.I.Ch.E. J., 5, pp174177.

Harrop, J.A. and Stenhouse, J.I.T. (1969) Theoretical predictions of inertial impaction efficiency in filters, Chem. Eng. Sci., 24, pp1475-1489.

Hildyard, M.L., Ingham, D.B., Heggs, P.J. and Kelmanson, M.A. (1985) Integral equation solution of viscous flow through a fibrous filter, In Boundary Elements VII (ed. C.A.Brebbia and G.Maier), Springer-Verlag, Berlin.

Ingham, D.B., Hildyard, M.L. and Heggs, P.J. (1989) The particle collection efficiency of a cascade pf cylinders, The Canadian J. of Chem. Eng., 67, pp545-553. Japuntich, D.A., Stenhouse, J.I.T. and Liu, B.Y.H. (1994) Experimental results of solid monodisperse particle clogging of fibrous filters, J.Aerosol Sci., 25, pp385-393. Jaswon, M.A. and Symm, G.T. (1977) Integral equation methods in potential theory and elasostatics, Academic Press, London.

Jung, Y. and Tien, C. (1993) Simulation of aerosol deposition in granular media, Aerosol Sci. and tech., 18, pp418-440. 
Kanaoka, C., Emi, H. and Tanthapanichakoon, W. (1983) Convective diffusional deposition and collection efficiency of aerosol on a dust-loaded fibre, A.I.Ch.E., 29, pp895-902

Karadimos, A. and Ocone, R. (2003) The effect of the flow field recalculation on fibrous filter loading: a numerical simulation, Powder tech., 137, pp109-119.

Kelmanson, M.A. (1983a) Boundary integral equation solution of viscous flows with free surfaces, J. Eng. Math., 17, pp329-343.

Kelmanson, M.A. (1983b) An integral equation method for the solution of singular slow flow problems, J. Comp. Phy., 51, pp139-158.

Konstandopoulos, A.G., Kostoglou, M. and Rosner, D.E. (1998) Shape evolution of particulate deposits growing on cylinders in cross flow, World Congress on Particle Technology 3, Brighton, U.K., 284/1-284/12

Kostoglou, M. and Konstandopoulos, A.G. (2000) Particulate deposit shape evolution on cylinders in cross-flow at high Stokes numbers, J. Aerosol Sci., 31, pp427-436. Kuwabara, S. (1959) The forces exerted by randomly distributed parallel circular cylinders or spheres in a viscous flow at small Reynolds numbers, J. Phy. Soc. Japan, 14, pp527-532.

Lee, K.W. and Gieseke, J.A. (1980) Note on the approximation of interceptional collection efficiencies, J.Aerosol Sci., 11, 335-341.

Payatakes, A.C. (1977) Model of transient aerosol particle deposition in fibrous media with dendritic pattern, A.Ch.E.J., 23, pp192-202.

Payatakes, A.C. and Gradon, L. (1980) Dendritic deposition of aerosols by convective Brownian motion for small, intermediate and high Knudsen numbers, A.Ch.E.J., 26, pp443-454.

Pich, J. (1973) Theory of gravitational capture of particles in fibrous aerosol filters, Aerosol Sci., 4, pp217-226.

Przekop,R., Moskal, M., and Gradon, L. (2003) Lattice-Boltzmann approach for description of the structure of deposited particulate matter in fibrous filters, J. Aerosol Sci., 34, pp133-147

Rao, N. and Faghri, M. (1988) Computer modelling of aerosol filtration by fibrous filters, Aerosol Sci. and tech., 8, pp133-156.

Stechkina, I.B. and Fuchs, N.A. (1966) Studies on fibrous aerosol filters -I.

Calculations of diffusional deposition of aerosols in fibrous filters, Ann. Occup. Hyg., 9, pp59-64. 
Stenhouse, J.I.T., Japuntich, D.A. and Liu, B.Y.H. (1992) The behaviour of fibrous filters in the initial stages of filter loading, J.Aerosol Sci., 23, S761-764.

Stenhouse, J.I.T. and Trottier, R.A. (1991) The loading of fibrous filters with submicron particles, J.Aerosol Sci., 22, S777-S780.

Thomas, D., Penicot, P., Contal, P., Leclerc, D. and Vendel, J. (2001) Clogging of fibrous filters by solid aerosol particles, Experimental and modelling study, Chem. Eng. Sci., 56, pp3549-3561.

Zhu, C., Lin, C.H. and Cheung, C.S. (2000) Inertial impaction-dominated fibrous filtration with rectangular or cylindrical fibers, Powder tech., 112, pp149-162. 
Figure captions

Figure 1 The domains considered. Fig 1a represents the Kuwabara cell and the dimensionless scaling replaces $d / 2$ by 1 and $b_{0}$ by $h_{1}=2 b_{0} / d$. In Fig. $1 b$ the cell represents a fibre within a system of parallel cylindrical fibres, and the dimensionless scaling replaces $\mathrm{d} / 2$ by 1 and $\mathrm{h}$ by $\mathrm{h}_{2}=2 \mathrm{~h} / \mathrm{d}$.

Figure 2 The interception radius for the particles, $r_{1}$, is $d / 2+r_{p}$ which is replaced by $1+\kappa$ in dimensionless units.

Figure 3 Boundaries between regions in the $\kappa-\delta$ plane where deposition at $\theta=0$ is specified by a different integral and will have different characteristics. Points corresponding to the physical filter cases shown in Table 1 are shown with the exception of case 1 where they are very close to those of case 8 . The region below the lower line corresponds to the lower small $\delta$ region and the region above the upper line to the upper large $\delta$ region where analytical results are obtained in the text.

Figure $4 \eta_{\text {SF }}$ as a function of Pe for $\alpha=0.05, \kappa=0.05, \kappa=0.1$ and $\kappa=0.2$.Also shown are the results of Stechkina and Fuchs (1966).

Figure 5. $\eta$ as a function of Pe for $\kappa=0.0025, \alpha=0.01, \alpha=0.05$ and $\alpha=0.1$.

Figure 6. $\partial \mathrm{n} / \partial \mathrm{N}$ as a function of $\theta$ using the 3 different flow models. Also shown are the results from the model of Friedlander (1977). In 6a Pe=5891, $\kappa=0.0025$, in 6b $\mathrm{Pe}=363114, \kappa=0.05$.

Figure $7 \frac{\partial \mathrm{n}}{\partial \mathrm{N}}$ as a function of $\theta$ determined numerically and using equation (40) for (a) $\mathrm{Pe}=1473, \kappa=0.05$, (b) $\mathrm{Pe}=5891, \kappa=0.0025$, (c) $\mathrm{Pe}=14728, \kappa=0.005$

Figure $8 \frac{\partial \mathrm{n}}{\partial \mathrm{N}}$ as a function of $\theta$ for $\mathrm{Pe}=5891, \kappa=0.0025$ 
Figure 9. Evolution of the deposit on the fibre surface for $P e=5891, \kappa=0.0025$. Flow is from left to right.

Figure 10. $\eta$ as a function of Pe for packing fraction, $\alpha=0.01, \alpha=0.05$ and $\alpha=0.1$ for no deposit and for 1 layer of deposit on the fibre.

Figure 11. $\eta$ as a function of $\alpha$ for $P e=5891, \kappa=0.0025$ with no deposit and with 1 layer of deposit. 
Table 1. Parameters for cases considered. All cases are for atmospheric pressure at 20C and $\alpha=0.05$

\begin{tabular}{|c|c|c|c|c|c|c|c|c|}
\hline \multirow{3}{*}{$\begin{array}{l}\text { Case } \\
\text { no. }\end{array}$} & Fibre & Velocity & $\mathrm{Re}$ & \multirow{3}{*}{$\begin{array}{c}\text { Particle } \\
\text { radius } \\
\mathrm{r}_{\mathrm{p}} \mathrm{nm}\end{array}$} & \multirow[t]{3}{*}{$\mathrm{Pe}$} & \multirow[t]{3}{*}{$\kappa=2 r_{p} / d$} & \multirow{3}{*}{$\begin{array}{r}\text { delta } \\
\text { k parallel }\end{array}$} & \multirow{3}{*}{$\begin{array}{l}\delta \\
\mathrm{k} \text { isolated }\end{array}$} \\
\hline & Diameter & & & & & & & \\
\hline & $\mathrm{d} \mu \mathrm{m}$ & $\mathrm{U}_{0} \mathrm{~m} / \mathrm{s}$ & & & & & & \\
\hline 1 & 20 & 0.5 & 0.6595 & 50 & 7364 & 0.005 & 0.06129 & 0.08690 \\
\hline 2 & 20 & 0.5 & 0.6595 & 100 & 22600 & 0.01 & 0.04218 & 0.05980 \\
\hline 3 & 20 & 0.5 & 0.6595 & 500 & 363100 & 0.05 & 0.02106 & 0.02986 \\
\hline 4 & 2 & 0.5 & 0.06595 & 50 & 1473 & 0.05 & 0.13205 & 0.23402 \\
\hline 5 & 2 & 0.5 & 0.06595 & 100 & 4519 & 0.1 & 0.09087 & 0.16104 \\
\hline 6 & 2 & 0.5 & 0.06595 & 500 & 36310 & 0.5 & 0.04537 & 0.08041 \\
\hline 7 & 40 & 0.1 & 0.2638 & 50 & 5891 & 0.0025 & 0.08319 & 0.13128 \\
\hline 8 & 40 & 0.1 & 0.2638 & 100 & 1808 & 0.005 & 0.05725 & 0.09034 \\
\hline 9 & 40 & 0.1 & 0.2638 & 500 & 14525 & 0.025 & 0.02858 & 0.04511 \\
\hline
\end{tabular}




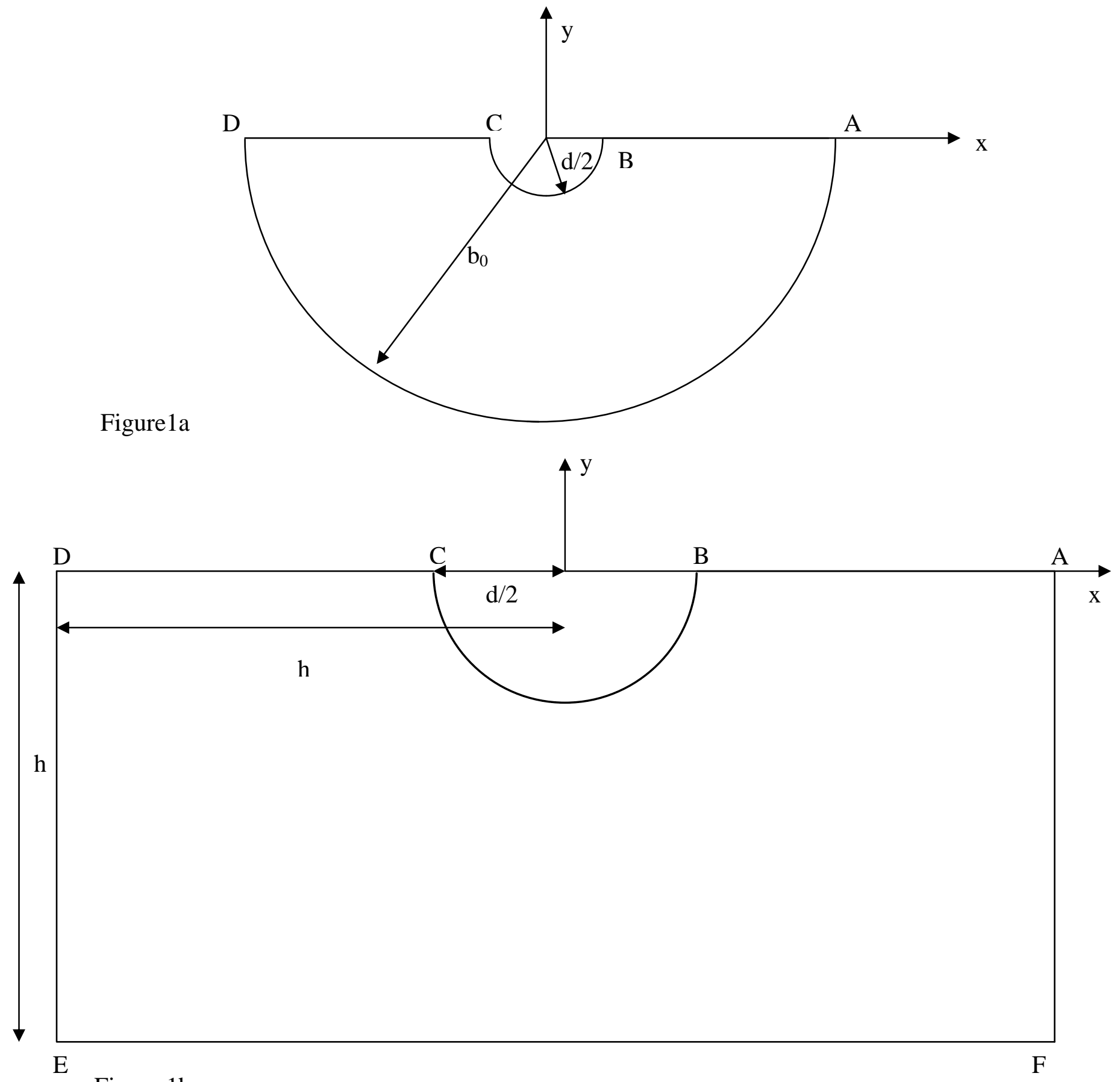

Figure 1b 
Figure 2. Error!
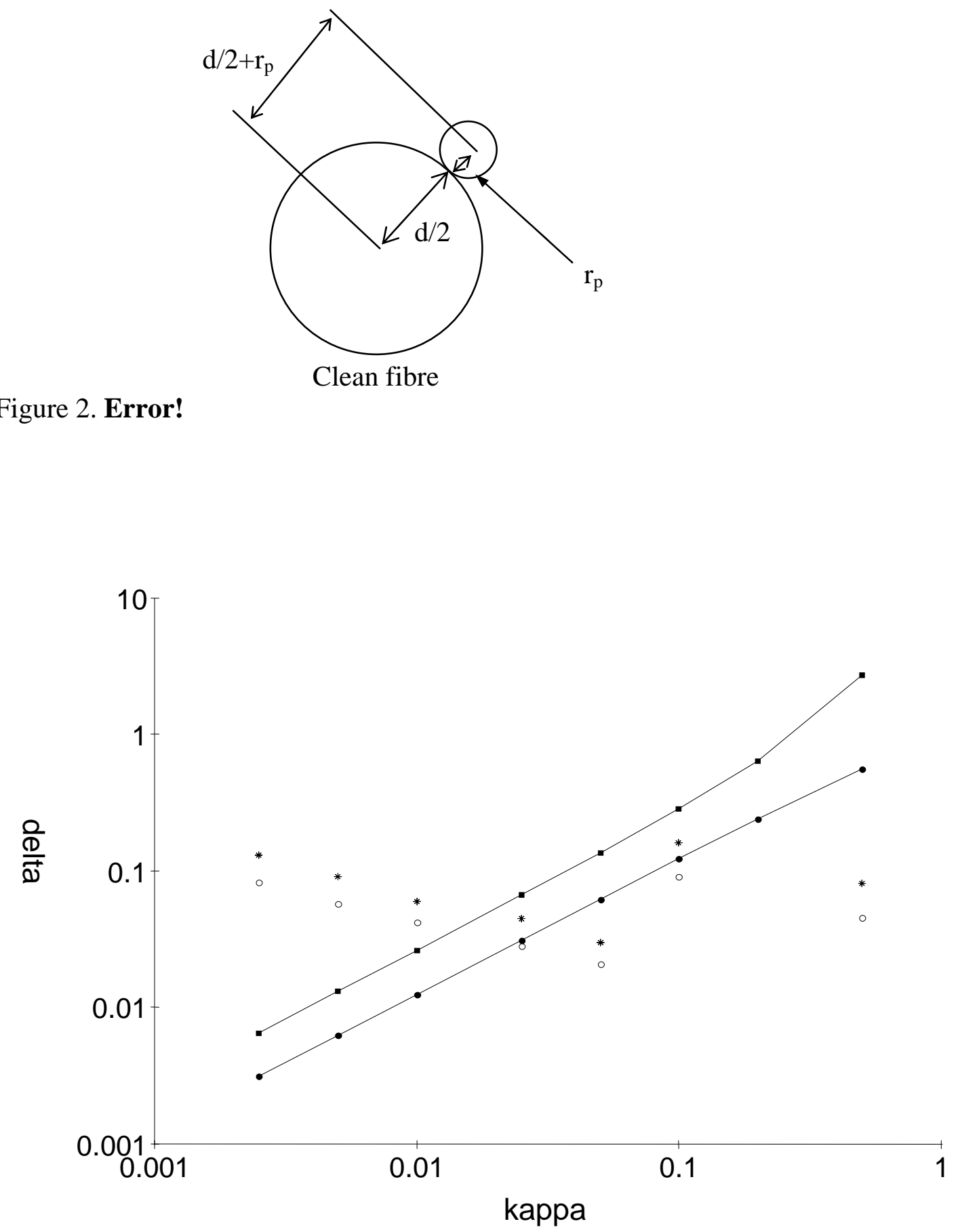

- delta $A_{\kappa}-B_{\kappa} \quad-$ delta $B_{\kappa}-C_{\kappa}$

* delta isolated $\circ$ delta parallel

Figure 3 


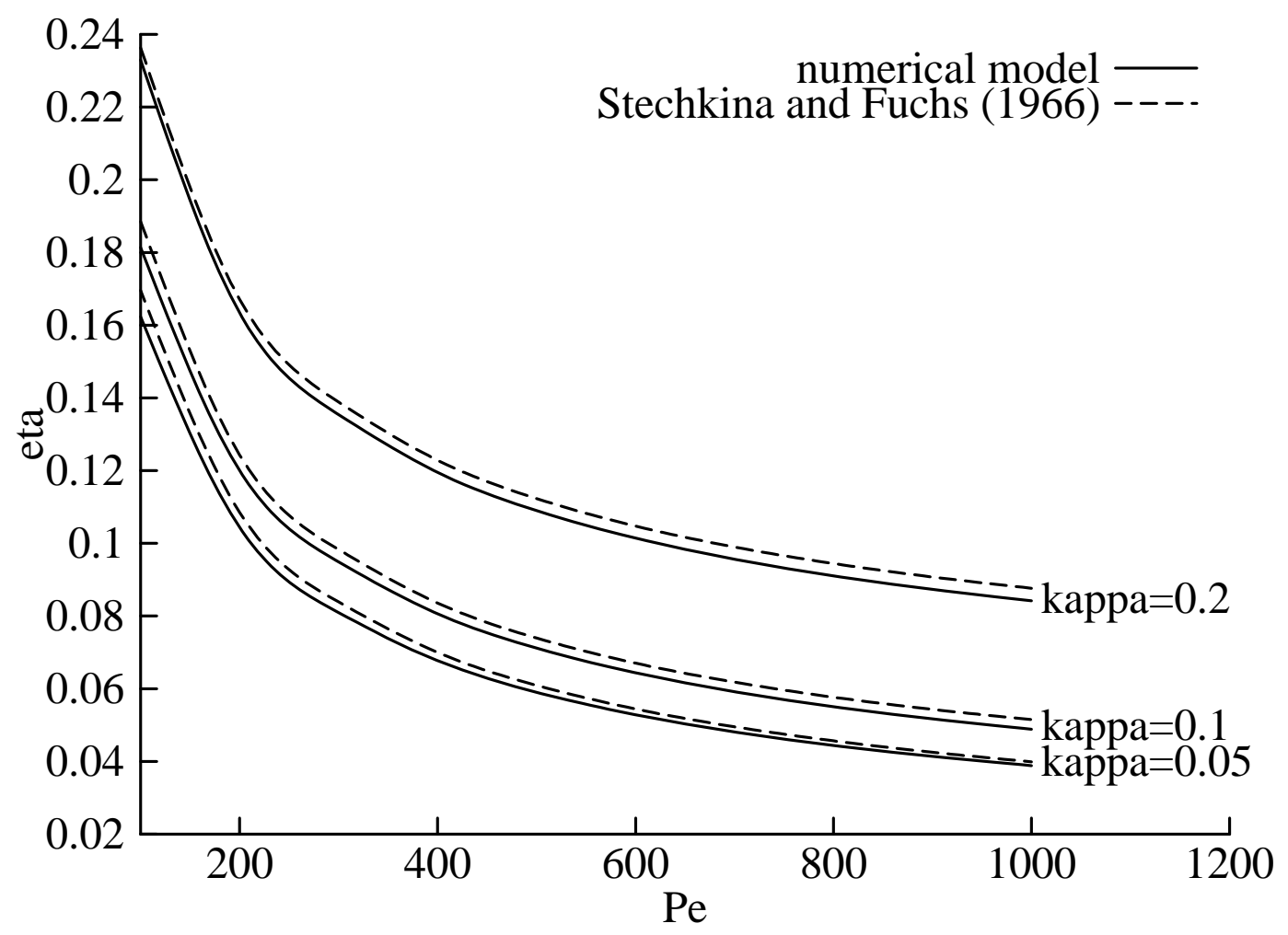

Figure 4

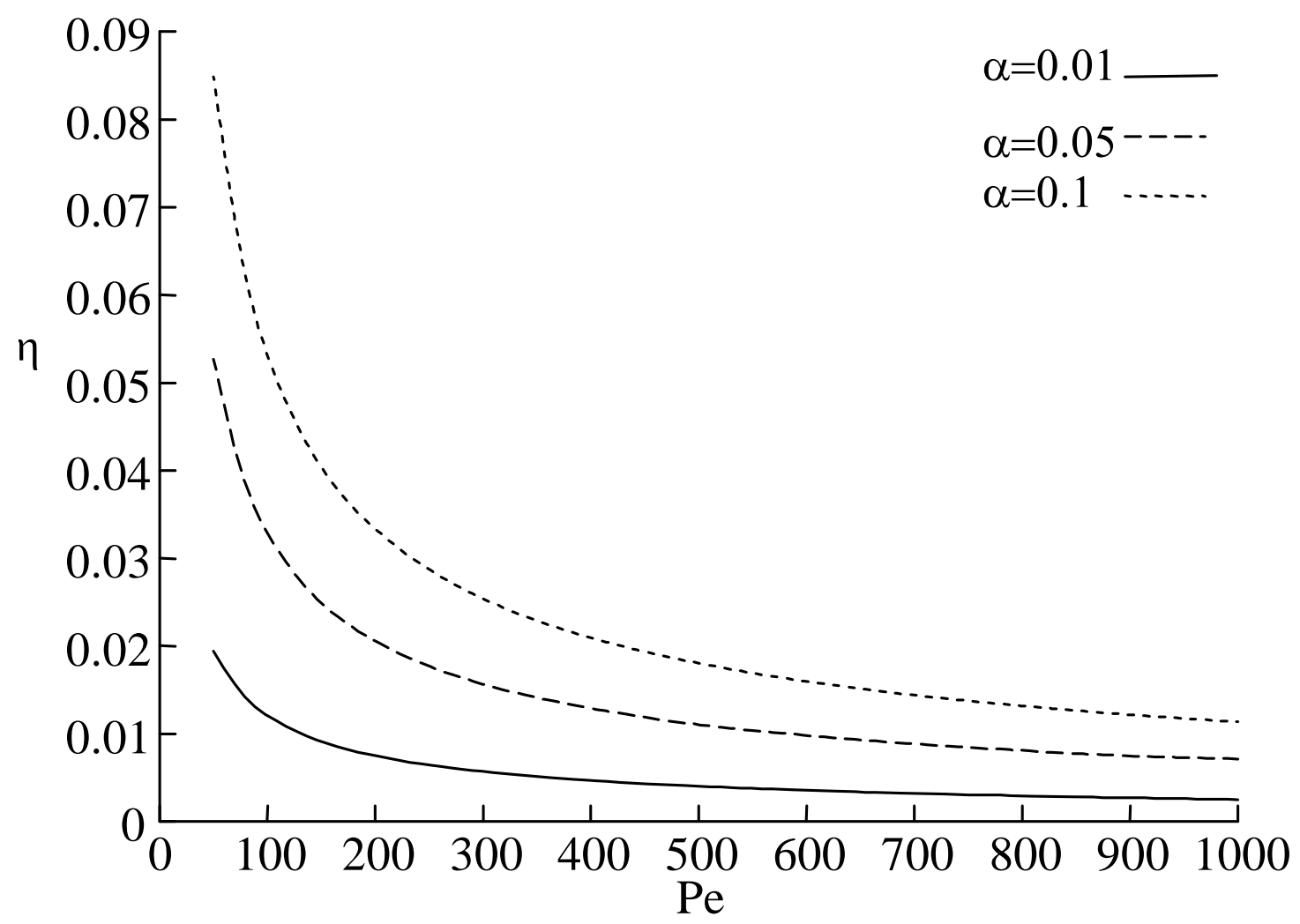

Figure 5. 


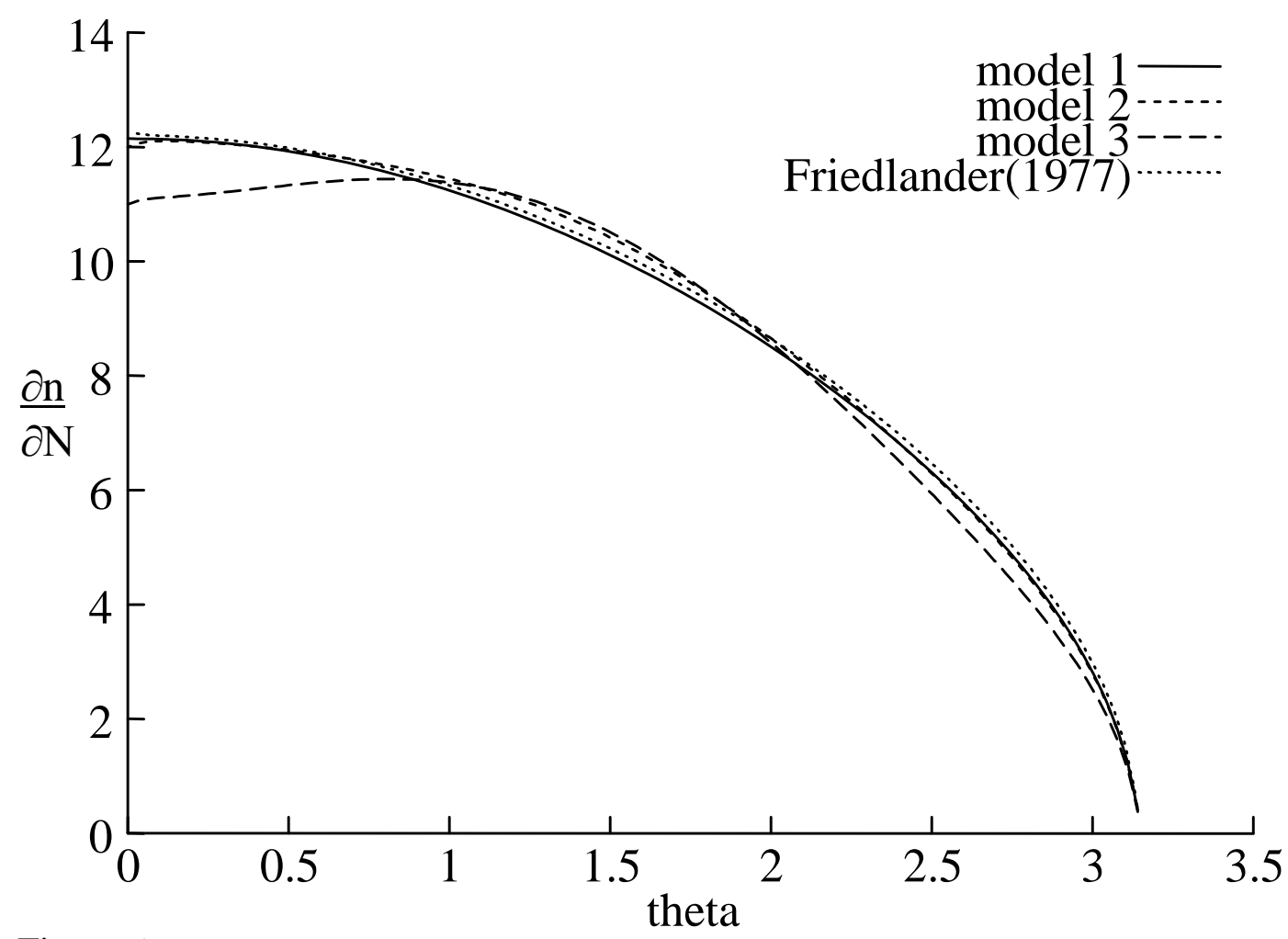

Figure 6a

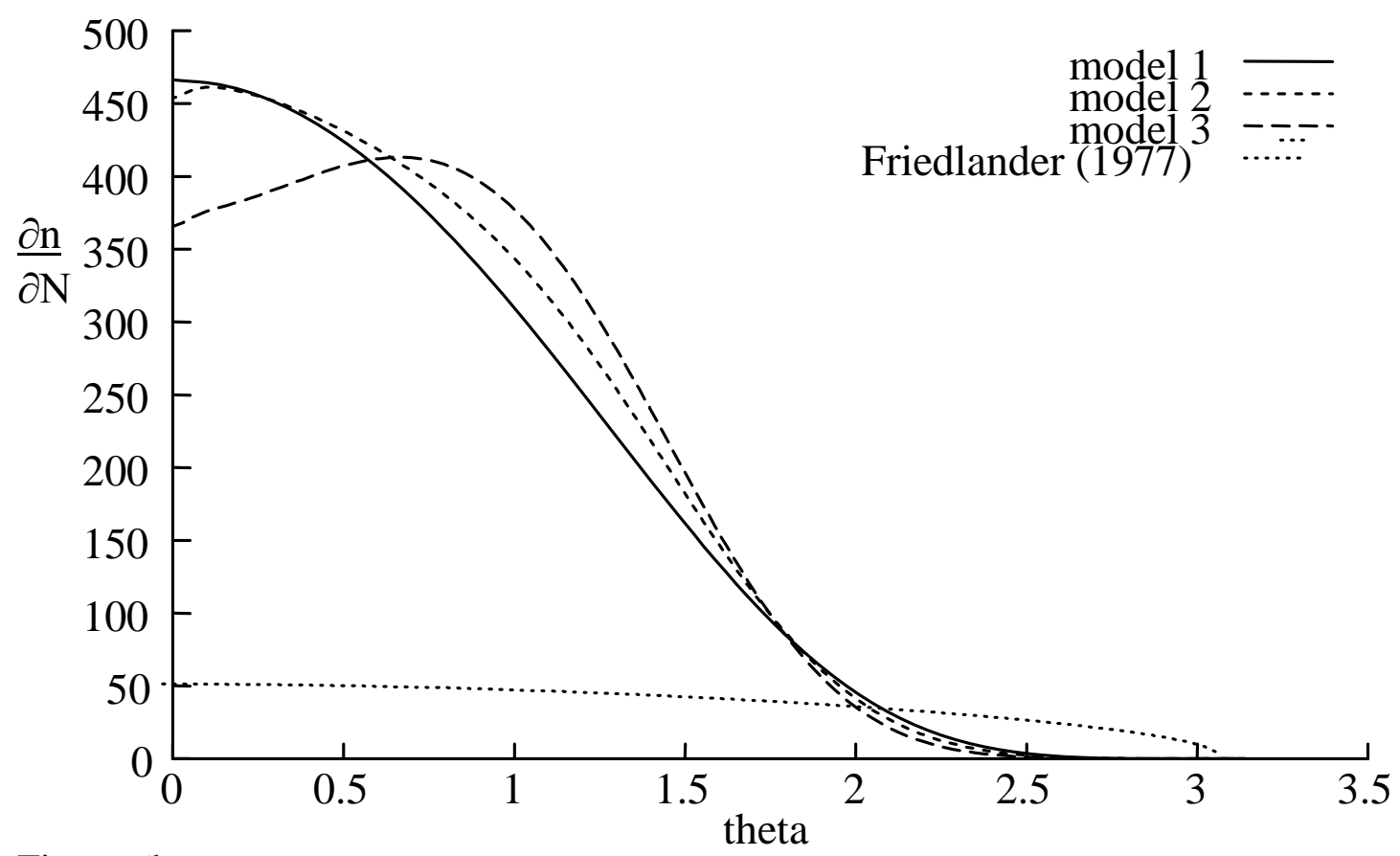

Figure 6b 


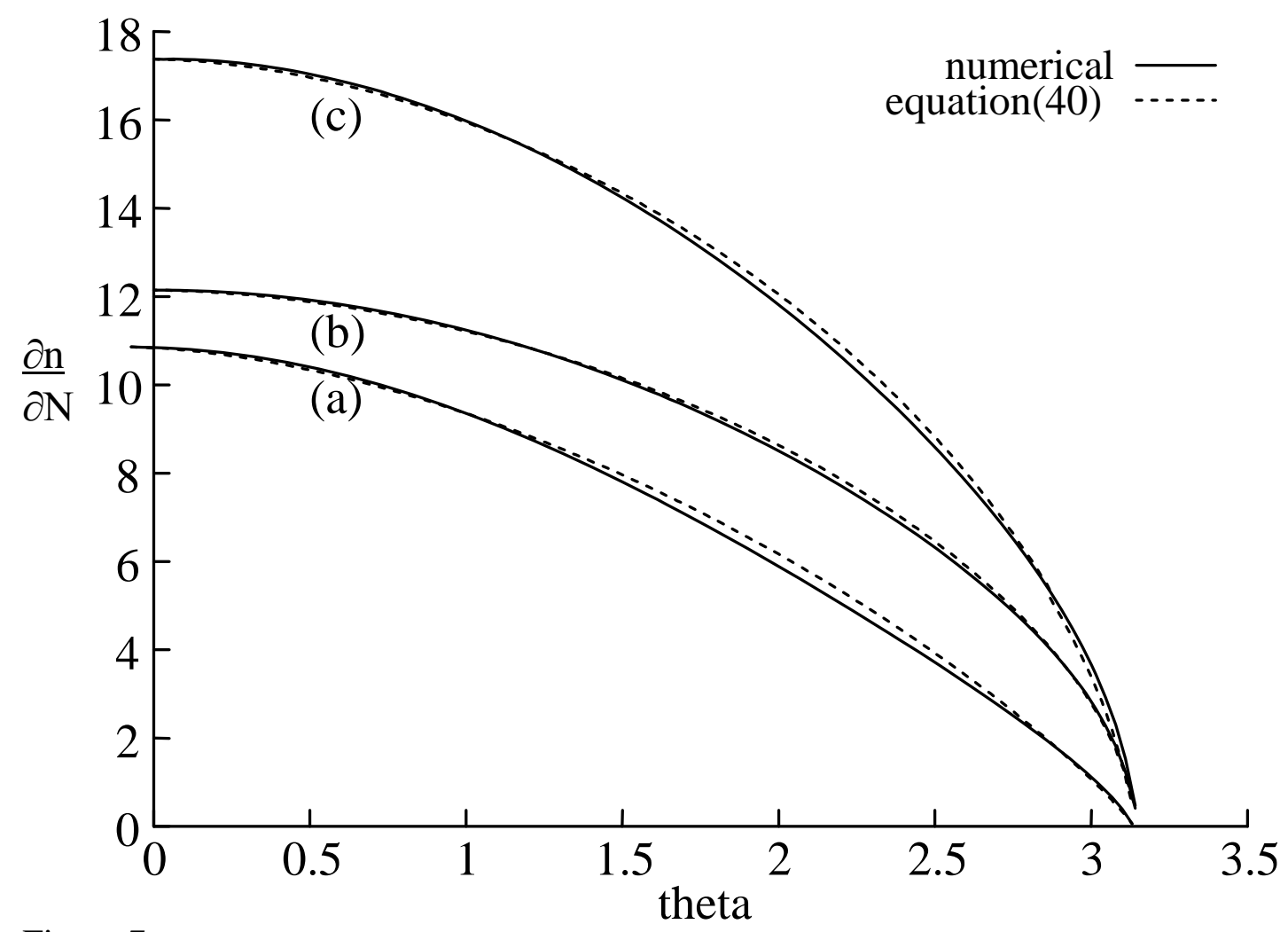

Figure 7

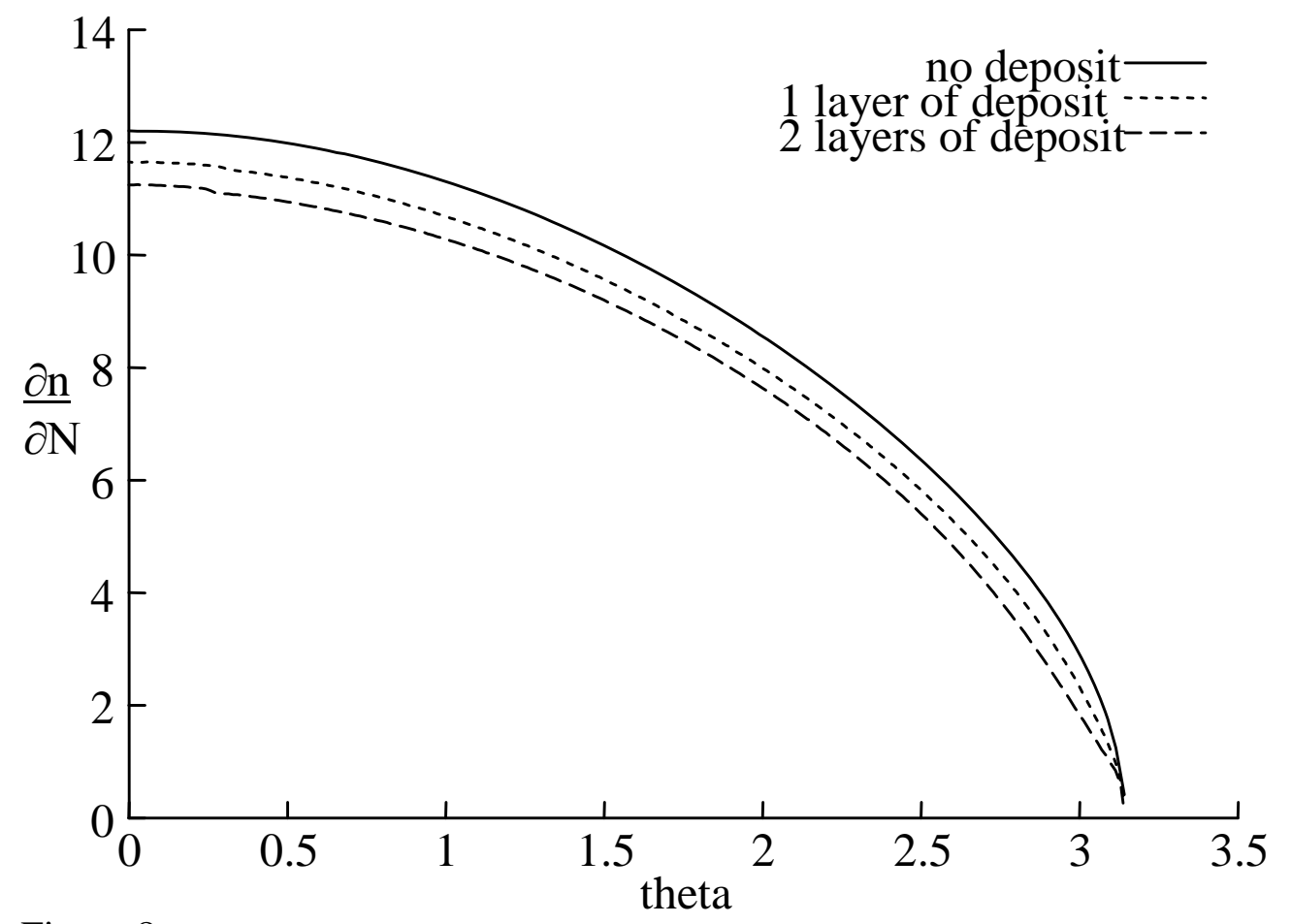

Figure 8 


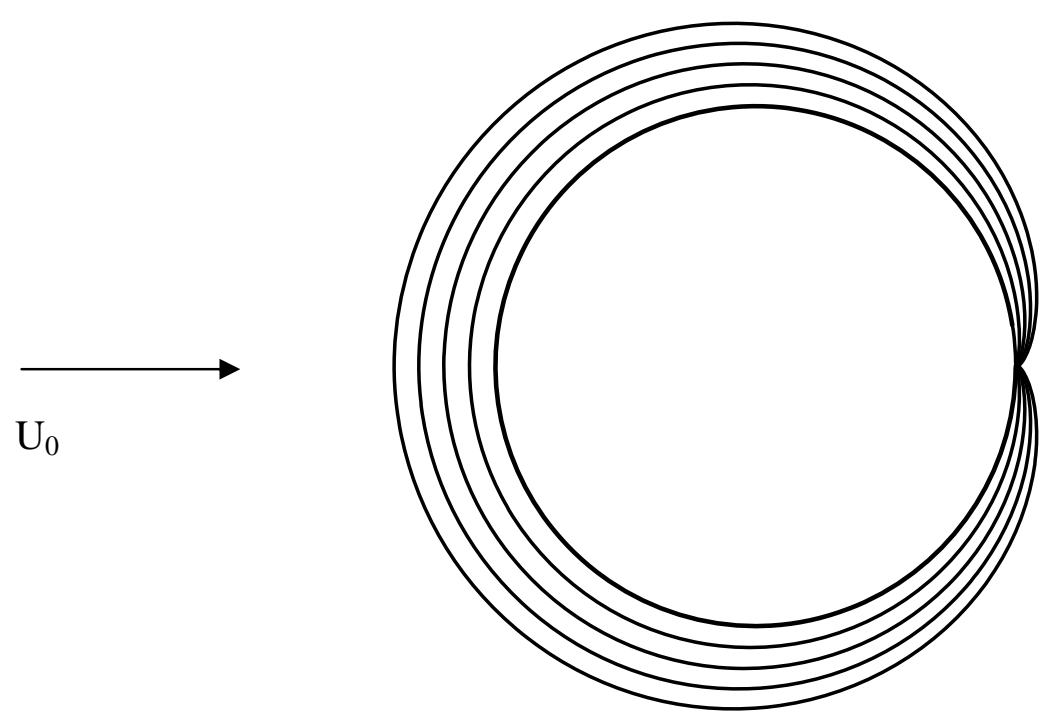

Figure 9.

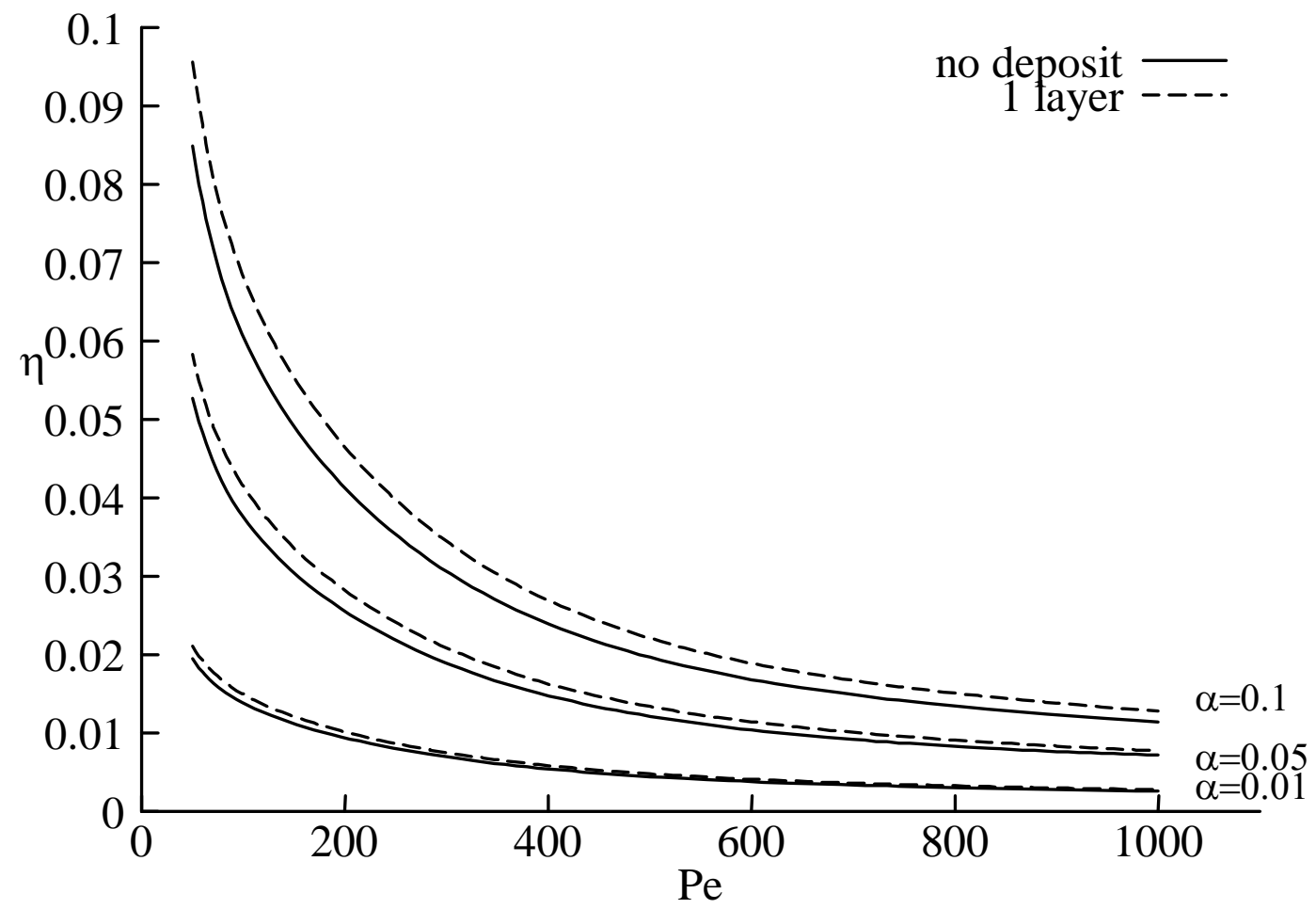

Figure 10. 


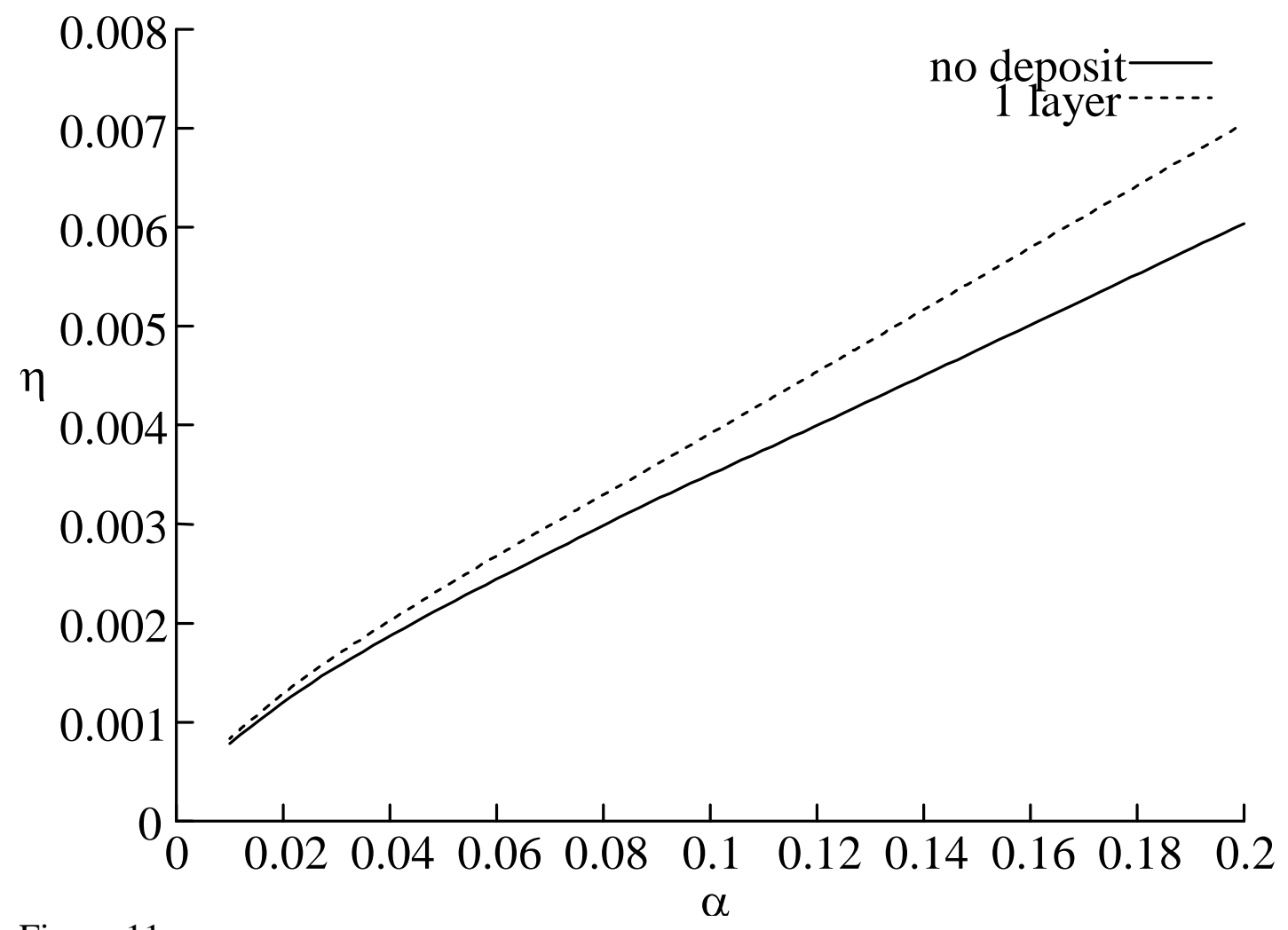

Figure 11. 